\title{
Frontal Cortex Activation Causes Rapid Plasticity of Auditory Cortical Processing
}

\author{
Daniel E. Winkowski, ${ }^{1 \star}$ Sharba Bandyopadhyay, ${ }^{1,2 \star}$ Shihab A. Shamma, ${ }^{1,3}$ and Patrick 0. Kanold ${ }^{1,2}$ \\ ${ }^{1}$ Institute for Systems Research, ${ }^{2}$ Department of Biology, and ${ }^{3}$ Department for Electrical and Computer Engineering, University of Maryland, College Park, \\ Maryland 20742
}

Neurons in the primary auditory cortex (A1) can show rapid changes in receptive fields when animals are engaged in sound detection and discrimination tasks. The source of a signal to A1 that triggers these changes is suspected to be in frontal cortical areas. How or whether activity in frontal areas can influence activity and sensory processing in A1 and the detailed changes occurring in A1 on the level of single neurons and in neuronal populations remain uncertain. Using electrophysiological techniques in mice, we found that pairing orbitofrontal cortex $(\mathrm{OFC})$ stimulation with sound stimuli caused rapid changes in the sound-driven activity within $\mathrm{A} 1$ that are largely mediated by noncholinergic mechanisms. By integrating in vivo two-photon $\mathrm{Ca}^{2+}$ imaging of $\mathrm{A} 1$ with $\mathrm{OFC}$ stimulation, we found that pairing $\mathrm{OFC}$ activity with sounds caused dynamic and selective changes in sensory responses of neural populations in A1. Further, analysis of changes in signal and noise correlation after OFC pairing revealed improvement in neural population-based discrimination performance within A1. This improvement was frequency specific and dependent on correlation changes. These OFC-induced influences on auditory responses resemble behavior-induced influences on auditory responses and demonstrate that OFC activity could underlie the coordination of rapid, dynamic changes in $\mathrm{A} 1$ to dynamic sensory environments.

\section{Introduction}

Cognitive flexibility is vital in dynamic sensory environments to achieve one's goals. Such flexibility requires dynamic modulation of sensory processing to adjust for ambient changes (Kastner and Pinsk, 2004; Reynolds and Chelazzi, 2004; Gilbert and Sigman, 2007; Fritz et al., 2007a; Meyer, 2011). For example, while discriminating between sounds or while detecting tones from noise, neuronal responses in auditory cortex (A1) change (Fritz et al., 2003, 2007b). For A1 neurons to alter responses during behavior, A1 must receive a signal of when to change and then A1 circuits adjust. How such changes are initiated, and how stimulus-driven activity in individual A1 neurons and across populations of A1 neurons are adjusted remains uncertain.

Activity in neuromodulatory or limbic regions can alter neural responses and behavior (Kilgard and Merzenich, 1998; Bao et al., 2001; Froemke et al., 2007; Weinberger, 2007; Chavez et al., 2009; Froemke et al., 2013). For example, stimulation of nucleus basalis (NB) causes a decorrelation of sensory-evoked activity in visual and auditory cortex (Bakin and Weinberger, 1996; Goard and Dan, 2009), which can enhance sensory encoding (Averbeck

\footnotetext{
Received Jan. 14, 2013; revised Sept. 20, 2013; accepted 0ct. 7, 2013.

Author contributions: D.E.W., S.B., S.A.S., and P.O.K. designed research; D.E.W. and S.B. performed research; D.E.W. and S.B. analyzed data; D.E.W., S.B., S.A.S., and P.O.K. wrote the paper.

This work is supported by National Institute on Deafness and Other Communication Disorders grants R01DC009607 (P.0.K.) and R01DC005779, Advanced ERC 295603 (S.A.S.), and AFOSR DURIP (S.A.S. and P.0.K.). We thank Hey-Kyoung Lee, Dan Butts, and Matt Roesch, and Robert Froemke for valuable comments on earlier versions of this manuscript and Stephen David, Paul Watkins, and llana Witten for helpful discussions.

${ }^{*}$ D.E.W. and S.B. contributed equally to this work.

Correspondence should be addressed to Patrick 0. Kanold, Department of Biology, University of Maryland, 1116 Biosciences Research Building, College Park, MD 20742. E-mail: pkanold@umd.edu.

DOI:10.1523/JNEUROSCI.0180-13.2013

Copyright $\odot 2013$ the authors $\quad 0270-6474 / 13 / 3318134-15 \$ 15.00 / 0$
}

et al., 2006; Goard and Dan, 2009; Josić et al., 2009). Moreover, stimulation of ventral tegmental area (VTA) or basolateral amygdala can also alter A1 responses (Bao et al., 2001; Chavez et al., 2009). All these regions receive input from frontal cortex (FC; Cavada et al., 2000; Ghashghaei and Barbas, 2002; Golmayo et al., 2003; Miyashita et al., 2007; Budinger et al., 2008). Because FC is active in auditory behavior when changes in $\mathrm{Al}$ occur, and since signals controlling changes in sensory responses as a consequence of behavioral demands might originate in FC, the FC might signal task-specific information to A1 (Reynolds and Chelazzi, 2004; Wallis, 2007; Fritz et al., 2007a, 2010).

Here, we investigate whether FC activation can cause changes in A1. While prior studies observed response plasticity of single A1 neurons using tone overexposures, behavior, NB microstimulation, VTA microstimulation, or classical conditioning (Kilgard and Merzenich, 1998; Bao et al., 2001; Polley et al., 2006; Weinberger, 2007; Chavez et al., 2009), how populations of A1 neurons change in these experimental conditions is largely unknown. We use in vivo two-photon $\mathrm{Ca}^{2+}$ imaging of $\mathrm{A} 1$ responses in mice to measure how frequency selectivity and functional organization in populations of A1 layer 2/3 neurons were influenced by FC activity. Two-photon $\mathrm{Ca}^{2+}$ imaging offers advantages over traditional recording approaches used in previous studies of Al plasticity by allowing simultaneous monitoring of populations of individual neurons in a less biased manner. Thus, this approach allows the study of the population dynamics and encoding within local A1 networks as a result of signals originating within FC.

We show that frequency selectivity and functional organization in $\mathrm{A} 1$ neurons were influenced by pairing $\mathrm{FC}$ activation with tone presentation. In individual neurons, frequency selectivity changes were diverse showing increased or decreased responses 
to the presented tone. However, over the population, responses to the presented tone increased and signal and noise correlations changed suggesting altered intralaminar and interlaminar connectivity. Using information theoretic analyses we find increased discriminability of the presented tone. Thus, small diverse changes in individual neurons led to an increased population encoding performance.

\section{Materials and Methods}

All procedures were approved by the University of Maryland Institutional Animal Care and Use Committee.

\section{Animal preparation}

Mice (either sex, P20-P35, C57BL/6; The Jackson Laboratory) were prepared by inducing surgical levels of anesthesia with $2-3 \%$ isoflurane in $100 \%$ oxygen. Throughout all procedures, the animal's skin temperature was maintained at $33-35^{\circ} \mathrm{C}$ (which corresponds to a core temperature of $37^{\circ} \mathrm{C}$ ) with a heating plate. A plate for securing the head was attached to the skull. To access Al a $2 \mathrm{~mm}$ diameter craniotomy was performed above the auditory cortex (Bandyopadhyay et al., 2010; Winkowski and Kanold, 2013).

\section{Auditory stimulation}

Sound stimuli were presented to the ear contralateral to the imaging/ recording hemisphere. The ear canal was accessed by making an incision from the ventral side of the ear canal. Sound stimuli (13 sinusoidal amplitude modulated (SAM) tones, $4-32 \mathrm{kHz}$ or $8-64 \mathrm{kHz}, 0.25$ octaves apart, $\sim 60 \mathrm{~dB} \mathrm{SPL}, 1 \mathrm{~s}$ long, $5 \mathrm{~Hz}$ full depth modulation $4-5$ s interstimulus intervals (ISI), or broadband noise pips $200 \mathrm{~ms}$ long, $10 \mathrm{~ms}$ linear on and off ramps) were generated using custom written software in MATLAB (MathWorks) and a D/A board (National Instruments). Stimuli were anti-alias filtered (PD-AAF-18; United Electronics), attenuated using a TDT PA5 (Tucker Davis Technologies;TDT), generated using TDT EC1 calibrated speakers (driven with TDT ED1 drivers), and delivered through a tube attached to the ear canal. Each stimulus was repeated $8-15$ times for imaging experiments.

\section{Orbitofrontal cortex stimulation}

The orbitofrontal cortex (OFC) was selected as the stimulation location in the FC since OFC projects to primary visual and auditory cortices (Golmayo et al., 2003). To access the OFC a small craniotomy was performed above the OFC ( $2.5 \mathrm{~mm}$ rostral from bregma and $1 \mathrm{~mm}$ lateral from the midline) on the side ipsilateral to the imaging location in A1. A low impedance $(0.1 \mathrm{M} \Omega$ ) bipolar stimulating tungsten electrode (Microprobes) was advanced into the OFC and secured in place with superglue or dental cement. To activate the OFC, the OFC was electrically stimulated through the implanted bipolar stimulating electrodes. For pairing a monophasic $200 \mathrm{~Hz}$ pulse train (cathodal pulses, $0.2 \mathrm{~ms}$ pulse duration) was delivered to the OFC using a constant current stimulus isolation unit (Cygnus Technologies). Stimulation sites in the OFC were anatomically confirmed post hoc (see Fig. 1A).

\section{Electrophysiological recordings}

To identify changes in sound-evoked activity in A1 we performed electrophysiological recordings. Epoxy-coated tungsten microelectrode arrays ( 16 channels arranged in a $4 \times 4$ grid; Microprobe) were used to record local field potentials (LFPs) in A1. The array was advanced into A1 slowly to a depth corresponding to $\mathrm{L} 2 / 3(<\sim 300 \mu \mathrm{m}$ from the pial surface). The electrodes were allowed to settle for $>\sim 20 \mathrm{~min}$ before the experiment commenced. Wide-band neural signals $(0.7-8 \mathrm{kHz})$ were passed through a preamplifier (Plexon), digitized at $10 \mathrm{kHz}$, and stored for off-line analysis using custom software written in MATLAB. The structure of the electrophysiology OFC pairing experiments was the same as for the imaging experiments (detailed below) except for one minor change. To measure evoked LFP responses of A1 sites, we presented SAM tones that were $400 \mathrm{~ms}$ in duration rather than $1 \mathrm{~s}$ in duration, which was the case for imaging experiments. Pairing episodes for electrophysiology and imaging followed the same protocol. Specifically, we presented a $1 \mathrm{~s}$ duration SAM tone of a single frequency simultaneously with electrical stimulation of a single OFC site (monophasic pulses, $200 \mathrm{~Hz}, 0.2 \mathrm{~ms}$ pulse width). The paired frequency was randomly selected to be within the ranges of frequencies to which recording sites were responsive. This pairing paradigm was repeated one hundred times. After completion of the OFC-sound pairing episode, the evoked LFP responses of A1 sites were measured again using the same stimuli.

To analyze the LFP signals in A1 we first removed $60 \mathrm{~Hz}$ line noise and its second harmonic $(120 \mathrm{~Hz})$ from the recording using the rmlinesc function, available from the Chronux toolbox (http://www.chronux. org). The denoised data were then low-pass filtered at $200 \mathrm{~Hz}$ and downsampled at $1 \mathrm{kHz}$ using the resample function in MATLAB. Responsive channels (sites) were defined by $t$ test $(p<0.01)$ across all baseline $(100$ ms prestimulus) and all poststimulus sound presentation periods (100 $\mathrm{ms}$ ). This criterion is somewhat conservative given that some sound stimuli do not elicit an LFP response. Nonetheless, neural signals from all responsive channels were subjected to spectral analysis.

Spectral analysis was performed using multitaper spectral estimation implemented in the Chronux toolbox (Bokil et al., 2010). For each channel, the responses at the pairing frequency $(\mathrm{PF})$ were averaged across trials and the evoked power was calculated by subjecting this average waveform to time-frequency analysis. Evoked power contains the stimulus-locked part of the oscillations. Induced power was computed by first performing time-frequency analysis for each trial, averaging the resulting spectra, and then subtracting the evoked power. This procedure removes stimulus-locked contributions to the oscillations from the spectral profile.

Spectrograms were computed using a moving window of length 100 $\mathrm{ms}$ (shifted in $2 \mathrm{~ms}$ increments) and three tapers $(K=3)$, permitting a time-bandwidth product of $2(T W=2)$. To reveal the frequencies of induced power, we used the R-spectrogram (Henrie and Shapley, 2005). An R-spectrogram was computed by normalizing the induced power in the spectrogram at each frequency by the mean power in that frequency during a baseline window before stimulus onset $(500 \mathrm{~ms})$ and allows for comparison across channels and across experiments.

To quantify differences between R-spectra before and after OFCsound pairing episodes, the induced power between 30 and $80 \mathrm{~Hz}$ (gamma band) from stimulus onset to stimulus offset $(400 \mathrm{~ms})$ was averaged for each channel at the pairing frequency. The average gamma power at PF before and after the pairing episode for each condition was compared. To compare across pharmacological conditions and account for any changes or differences in baseline activity, the effect of OFC pairing was compared using a desynchronization index [(post - pre)/ (post + pre) $)$.

\section{OFC inactivation}

To test if OFC activity is required the OFC was inactivated pharmacologically. OFC was accessed via a small craniotomy as detailed above. A glass pipette (tip diameter: $\sim 20 \mu \mathrm{m}, \sim 0.1 \mu \mathrm{l} / \mathrm{min}$ ) was lowered into the OFC. A mixture of GABA agonists ( $5 \mu \mathrm{g} / \mu \mathrm{l}$ muscimol and $2 \mu \mathrm{g} / \mu \mathrm{l}$ baclofen in artificial CSF (ACSF); Sigma) or vehicle (ACSF) was slowly pressure injected into the OFC via a microinjector (Nanoject II; Drummond Scientific) coupled to the glass pipette. The glass pipette was left in place for $>5$ min before implanting the stimulation electrode. To track the spread of the injected solution, rhodamine was included in the solutions and could be visualized post hoc to confirm overlap of injection and placement of stimulation electrode. In all cases, two injections (volume/injection $=250 \mathrm{nl}$; total volume $=500 \mathrm{nl}$ ) spaced $300 \mu \mathrm{m}$ apart vertically were made.

\section{A1 pharmacology}

To identify the involvement of the cholinergic system we pharmacologically blocked cholinergic receptors while recording LFPs in A1. The electrode array was advanced into $\mathrm{A} 1$ and acoustic responses were measured. Atropine (1 mM; to block muscarinic ACh receptors) or $3 \mathrm{~mm}$ mecamylamine (to block nicotinic ACh receptors) was applied to the cortical surface $(>\sim 45 \mathrm{~min})$ and left in place during the entire subsequent recording period as described in previous reports (Goard and Dan, 2009; Alitto and Dan, 2012). 
A
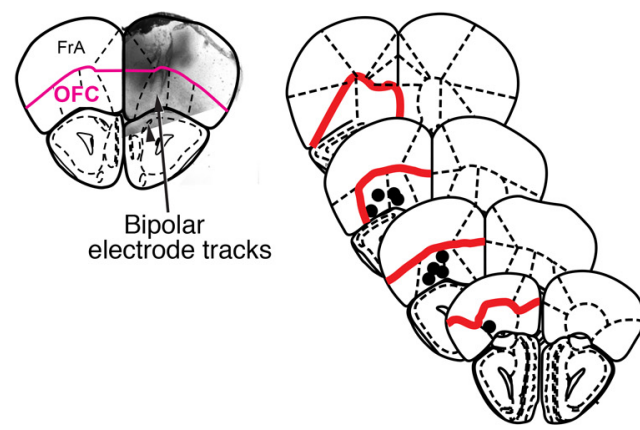

D
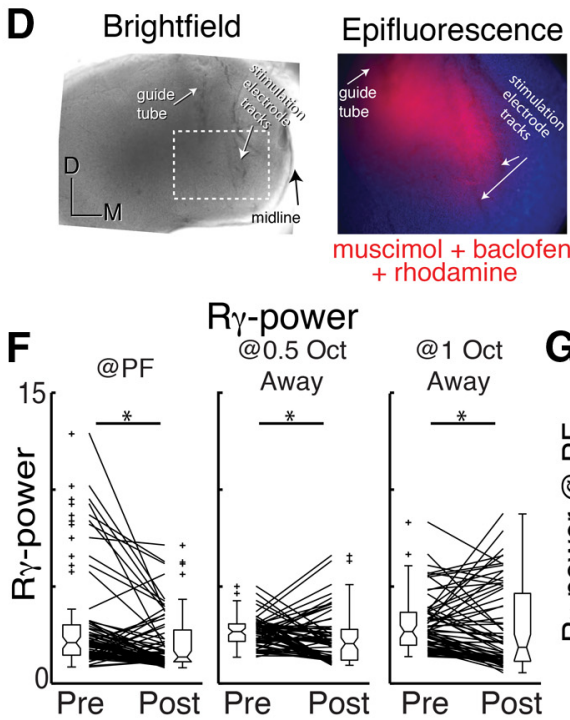

B

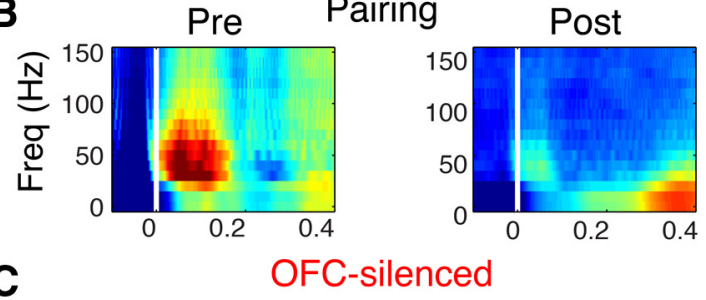

C

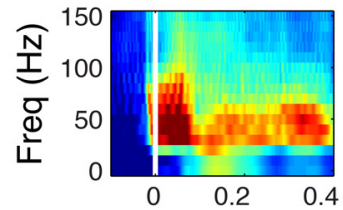

E

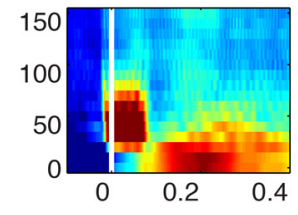

Saline
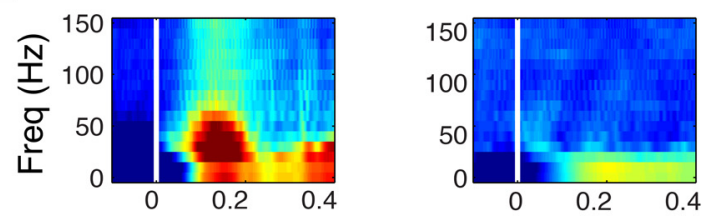

Time re. stimulus onset (sec)

G Silenced

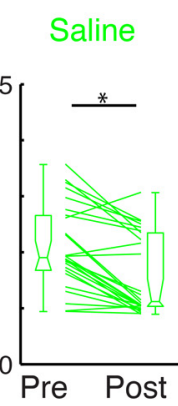

Hesynch
Index
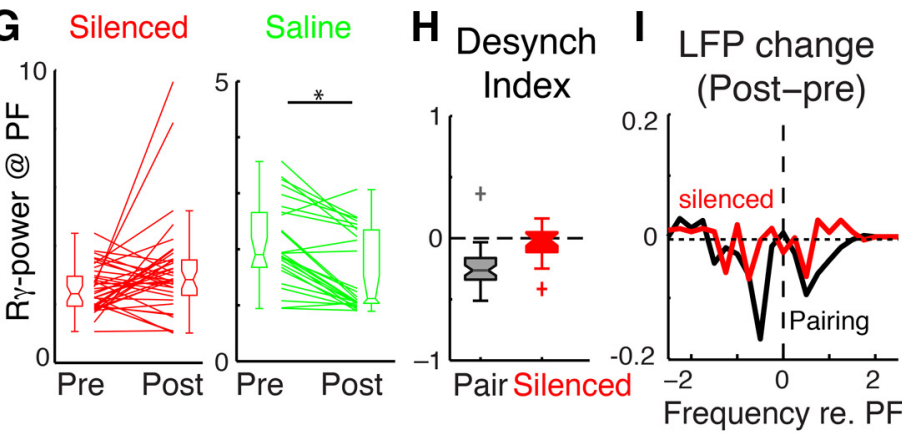

(octaves)

Figure 1. Response changes in $\mathrm{A} 1$ depend on $\mathrm{OFC}$ activation. $A$, Left, Bright-field image of a brain slice in the frontal cortex of the mouse showing the tracks of the bipolar stimulating electrodes used for OFC activation. Boundaries of OFC are indicated with a drawing from mouse brain atlas. Right, Mapping of stimulation locations in electrophysiological experiments. $\boldsymbol{B}$, Time-frequency analysis of ERPs at the PF before (left) and after (right) OFC-sound pairing episodes. R-spectra were computed for each responsive channel then averaged (see Materials and Methods). $C$, Same as in $\boldsymbol{B}$ and $C$ except that $\mathrm{OFC}$ was silenced with a mixture of GABA agonists before the OFC-sound pairing episodes. No change in power was observed. $\boldsymbol{D}$, Bright-field and fluorescence images showing locations of stimulation electrode and spread of muscimol in OFC. GABA agonist spread was tracked by rhodamine in the inactivating solution. The inactivated region encompasses the location of the stimulation electrode. $\boldsymbol{E}$, Same as in $\boldsymbol{B}$ and $($ except that ACSF was injected into the OFC before the OFC-sound pairing episodes. $\boldsymbol{F}, \mathbf{G}$, Comparison of average induced gamma ( $30-80 \mathrm{~Hz}$ ) power during the entire stimulus period ( $400 \mathrm{~ms}$ ) before (Pre) and after (Post) OFC-sound pairing episodes for each responsive channel under control conditions ( $\boldsymbol{F}$ ) or after application of GABA agonists or saline. $\boldsymbol{H}$, Desynchronization index. I, Difference in LFP peak amplitude tuning after pairing and with application of GABA agonists ("silenced").

\section{Two-photon imaging}

To monitor changes in the sound-evoked responses of single neurons in A1 we used in vivo two-photon imaging as described earlier (Bandyopadhyay et al., 2010; Winkowski and Kanold, 2013). A pipette $(8-10 \mu \mathrm{m}$ pulled tip) containing $\mathrm{Ca}^{2+}$-indicator dye Fluo-4 AM (Invitrogen) was advanced into the A1 (300-500 $\mu \mathrm{m}$ from the cortical surface). The dye solution was prepared by dissolving $50 \mu \mathrm{g}$ of dye in $4 \mu \mathrm{l}$ of $20 \%$ pluronic acid in dimethylsulfoxide (Invitrogen) and diluting (1:5-8) it with ACSF, containing either $100 \mu \mathrm{M}$ Alexa Fluor 594 (for visualization) or 20-50 $\mu \mathrm{M}$ SR-101 (for visualization and astrocyte identification). Once the pipette was in place, a series of pressure pulses (5-12 psi, PV830 Pneumatic PicoPump; WPI) were applied to inject the dye solution into the brain tissue. After loading of neurons was achieved (30-60 min post injection) the craniotomy was covered with warm agarose and coverslipped to create a stable imaging window. For imaging responses isoflurane anesthesia was reduced to $0.5-1 \%$. Sound-evoked $\mathrm{Ca}^{2+}$ responses were imaged at $256 \times 256$ resolution $(2 \times$ optical zoom) in small regions of interest (15-30 cells, $\sim 60-120 \mu \mathrm{m}$ side rectangular regions) with a two-photon microscope (Ultima; Prairie Technologies) coupled with a Spectra Physics Mai Tai two-photon laser $(800 \mathrm{~nm}$ or $810 \mathrm{~nm})$ and Prairie software. Images were collected at $\sim 10 \mathrm{~Hz}$ (average $105 \mathrm{~ms}$ frame period). A series of sequential images (15-25) was collected with the stimulus occurring at a certain frame number (7 or 10). Each stimulus was repeated $8-15$ times (usually 10). At the conclusion of some experiments, DiI crystals were inserted into the imaging site with a 26 gauge needle. The brain was removed and was stored in $4 \%$ paraformaldehyde at $38^{\circ} \mathrm{C}$ for $>3$ weeks and slices were cut to confirm labeling in the ventral division of the medial geniculate body.

The analysis of two-photon imaging data was performed as described earlier (Bandyopadhyay et al., 2010; Winkowski and Kanold, 2013). Individual cells were identified manually from average images of all repeats. The fluorescence of cells was measured in circular regions (3-5 pixel radius, 3.5-6 $\mu \mathrm{m}$ ) over the cell soma. Following a baseline period (3-6 frames), the stimulus was presented for $1 \mathrm{~s} \mathrm{(} \sim 9$ frames). The mean fluorescence was converted to $\Delta F / F$ by measuring mean fluorescence changes with respect to mean baseline fluorescence from multiple repeats of stimuli. The mean was obtained from bootstrap resampling from multiple repeats. Repeating the calculations by computing $\Delta F / F$ from each repeat and computing the mean did not change the mean effects. The $\Delta F / F$ for individual cells over the $1 \mathrm{~s}$ stimulus period for different frequencies is used for all analysis. Ninety-five percent confidence intervals of responses $(\Delta F / F)$ means were obtained from the bootstrap resampling 
of the multiple repeats. Tuning curves were obtained using the maximum significant $(p<0.05)$ response to each frequency during the stimulation period. Best frequency (BF) was defined as the peak (maximum) of the tuning curve. The tuning curves estimated from the overall mean response were similar in shape to those obtained from the peak response (median correlation between tuning curves computed in the two ways is 0.8 , for all tuning curves computed). The time and frequency response profiles were smoothed with a $2 \mathrm{D}$ Gaussian $(0.7$ pixel SD) and plotted as contour plots for representation. The differences in significant $(p<$ 0.05 ) responses before and after pairing were smoothed with the same Gaussian filter and then averaged across cells in a field or all cells for computing population mean effects.

Correlation of single cell changes with mean effect. To identify if response changes of individual neurons were similar, we computed the correlation of changes in individual neurons with the mean effect. We computed $2 \mathrm{~d}$ correlations between normalized (with $\|.\|_{2}$ norm) smoothed timefrequency response profiles $( \pm 1.5$ octaves around PF) of single neurons and the mean effect (see Fig. 6). The response differences in single neurons were shuffled in time and frequency using uniform random permutations. We then computed the correlations between the shuffled changes and the mean effect to obtain the shuffled distribution (Fig. 6A, gray distribution). We also computed these correlations by removing the one being correlated to compute the mean effect to remove bias of having the same template in the computation of the mean. This correction did not change any results indicating that the bias is negligible.

Signal and noise correlations. Correlations in response variability (noise correlations) and stimulus-related cross-correlations (signal correlations) in groups of neurons can have profound effects on population coding of sensory inputs and thus the functional organization of neural circuits (Averbeck et al., 2006; Josić et al., 2009; Cohen and Kohn, 2011). We therefore computed the changes in signal and noise correlations after OFC-sound pairing. Signal and noise correlations were computed as reported previously (Winkowski and Kanold, 2013). Briefly, normalized cross-correlations at 0 lag between the mean responses over nine stimulus frames of pairs of neurons to each stimulus were computed using MATLAB function xcorr with 0 and "coeff" as the parameters. The average across all stimuli for every pair was considered as the signal correlation between each pair of neurons. By removing time and considering only the tuning curves as responses and using the correlations between tuning curves produced the same results. Similarly, noise correlations were computed with the MATLAB function xcov at 0 lag and normalized by using coeff as the normalization parameter.

Sound discrimination analysis. To identify if the changes in neuronal responses can lead to changes in stimulus discrimination we computed stimulus discrimination performance based on a Kullback-Leibler $\left(D_{K L}\right)$ distance (Cover and Thomas, 2006). We calculated $D_{K L}$ in its symmetrical form $\left(D_{S}\right.$, Eqs. 1 and 3), which under our Gaussian assumption, is given below (Eqs. 2 and 3 ) where $\mu$ and $\Sigma$ represent the mean and covariance matrix of the Gaussian distributions.

$$
\begin{gathered}
D_{K L}(p \| q)=\int_{-\infty}^{\infty} p(x) \log \frac{p(x)}{q(x)} d x, \\
f \sim N\left(\mu_{f}, \sum_{f}\right) ; g \sim N\left(\mu_{g}, \sum_{g}\right), \\
D_{s}(f \| g)=D(f \| g)+D(g \| f) \\
=\frac{1}{2}\left[\left(\mu_{f}-\mu_{g}\right)^{T} \sum_{g}^{-1}\left(\mu_{f}-\mu_{g}\right)\right] . \\
D_{s}\left(r^{B} \| r_{+\Delta}^{B}\right)=\left(\mu^{B}-\mu_{+\Delta}^{B}\right)^{T} \sum^{B^{-1}}\left(\mu^{B}-\mu_{+\Delta}^{B}\right) .
\end{gathered}
$$

For a population of neurons (size $N$ ) the mean response to two frequencies $(\theta$ and $\theta+\Delta \theta)$ represented by $\mathbf{r}_{\theta}$ and $\mathbf{r}_{\theta+\Delta \theta}$ (vectors of size $N$ ) in the before $(B)$ or after $(A)$ condition is used to compute discrimination between the two stimuli as below (Eq. 4). The mean response is represented by $\mu$ and the covariance matrices $\left(\Sigma^{A}\right.$ and $\left.\Sigma^{B}\right)$ are assumed to be stimulus independent and computed from the average over all stimuli in
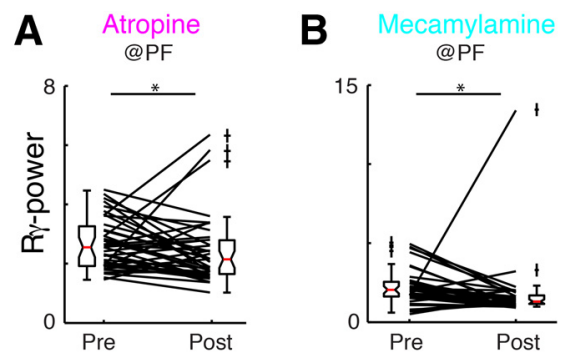

C Desynch Index
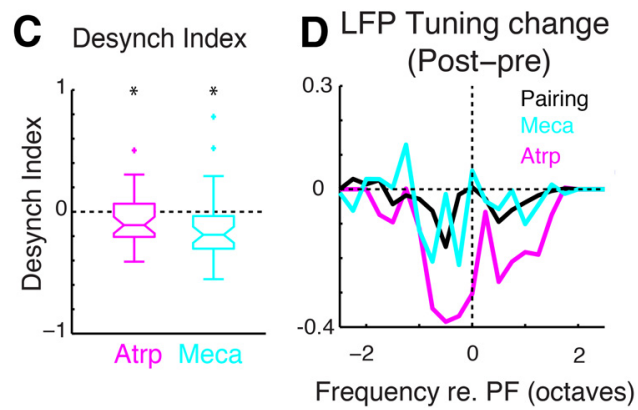

Figure 2. $\quad$ OFC stimulation-induced response changes in $\mathrm{A} 1$ are not mediated by cholinergic mechanisms. $\boldsymbol{A}, \boldsymbol{B}$, Comparison of average induced gamma $(30-80 \mathrm{~Hz})$ power during the entire stimulus period (400 ms) before (Pre) and after (Post) OFC-sound pairing episodes for each responsive channel after $A 1$ application of atropine $(\boldsymbol{A})$ or mecamylamine $(\boldsymbol{B}) . \boldsymbol{C}, \boldsymbol{D}$, Comparison of desynchronization indices $(\boldsymbol{C})$ and LFP peak tuning change $(\boldsymbol{D})$ across different pharmacological conditions (atropine application in A1, mecamylamine application in A1).

the after or before conditions. Relative change is assessed through $R_{A B}$ (Eq. 4)

$$
R_{A B}=\frac{\left(\mu^{A}-\mu_{+\Delta}^{A}\right)^{T} \sum^{A^{-1}}\left(\mu^{A}-\mu_{+\Delta}^{A}\right)}{\left(\mu^{B}-\mu_{+\Delta}^{B}\right)^{T} \sum^{B^{-1}}\left(\mu^{B}-\mu_{+\Delta}^{B}\right)}
$$

For the two groups discrimination performance was calculated as follows: for $\mathrm{PF} / \mathrm{O}$ group mean discrimination of $\mathrm{PF}$ and each of the other frequencies were computed; for $\mathrm{O} / \mathrm{O}$ group mean discrimination of the mean discrimination of each frequency (not PF) and each other non-PF frequency were computed, for every population size.

For the single cell $(N=1)$ case the discrimination performance for each of the single cells was calculated using Equation 4. The covariance matrix in this case was simply the variance for that cell. The first line of Equation 4 shows that, when assuming independence the covariance matrix becomes a diagonal matrix and hence discrimination for a population is the sum of discrimination by individual neurons, showing a linear dependence of discrimination on population size in the independent coding case. Thus median discrimination of two stimuli by a population of $N$ neurons is $N$ times median discrimination by the single neurons of that population.

For pairs of cells $(N=2)$ the discrimination performance by all possible pairs of cells were computed and median discrimination was computed. Since for larger population sizes $(N=3$ onward) the number of all possible $\mathrm{N}$-tuples in our population is usually extremely large, we bootstrapped by randomly choosing $N(3-14)$ cells from each field, as many times as the number of cell pairs in that field (for a field with $K$ cells, $N$ cells were chosen $K^{\star}(K-1) / 2$ times) and the median discrimination and interquartile range were computed. This process was repeated 10 times and median of the 10 medians along with the median of the 10 interquartile ranges are represented for all corresponding plots. We varied $N$ up to 14 , as beyond that there were progressively fewer imaging fields with $>14$ cells.

\section{Results}

\section{OFC pairing changes $\mathrm{A} 1$ population responses to} sound stimuli

To determine whether OFC activity could influence sensoryevoked Al activity, we performed electrophysiological experi- 
ments in which we recorded A1 activity before and after pairing stimulation of the OFC (a part of the FC) with sounds (Fig. $1 A, B)$. To sample A1 broadly, we used a 16 electrode 2D array and recorded sound-evoked, event-related potentials (ERPs) in superficial layers $(<\sim 300 \mu \mathrm{m}$ from surface), which allowed us to assess the population response with high temporal resolution. To investigate A1 changes with ERPs in both the temporal and spectral domains, we computed the induced power spectrum of the ERPs at PF before and after OFC-sound pairing episodes. Before OFC-sound pairing, ERPs evoked by the PF showed induced power across the gamma band $(20-100 \mathrm{~Hz})$ with a prominent peak near $30 \mathrm{~Hz}$ (Fig. 1B). After OFC pairing episodes, we found a striking decrease (median \pm iqr: $-22 \pm$ $30 \%)$ in gamma-band power at $\mathrm{PF}$ in nearly all cases $(n=68$ sites in five animals; $p<10^{-3}$; Wilcoxon sign rank; Fig. $1 B, F)$. Decreases in gamma-band power were also observed to sound stimuli away from the PF (Fig. 1F). The decrease in gamma-band power suggests a desynchronization of the population response. To compare our results across conditions, we computed a desynchronization index $\left(\left[\right.\right.$ post $_{\text {pairing }}-$ pre $\left._{\text {pairing }}\right) /\left(\right.$ post $_{\text {pairing }}+$ prepairing)]) for each recording channel. This analysis showed that OFC activation induced decrease in the desynchronization index (Pair: $-0.22 \pm 0.3$ median \pm iqr; $p<0.001$; Wilcoxon sign rank; Fig. $1 H$ ).

\section{OFC stimulation-induced changes in}

A1 require $\mathrm{OFC}$ activity

While electrical microstimulation activates OFC neurons, it can also potentially activate fibers of passage or cause antidromic activation of other structures, which, in principle, could underlie the observed changes in A1.

However, if activation of OFC neurons causes the decorrelation of A1 activity, then silencing OFC neurons during electrical stimulation should abolish the decorrelation of A1 neurons. To determine the degree to which this effect depended on the activation of OFC neurons, we pharmacologically silenced OFC neurons by injecting a mixture of GABA agonists $(5 \mu \mathrm{g} / \mu \mathrm{l}$ muscimol and $2 \mu \mathrm{g} / \mu \mathrm{l}$ baclofen) into the OFC before implanting the OFC stimulation electrode (Fig. 1C,D) and performed the experiment in the same way. Remarkably, with OFC neurons inactivated, induced gamma-band power was similar before and after OFC pairing episodes (median \pm iqr: $0.05 \pm 0.3 ; n=39$ sites in four animals; $p>$ 0.07; Wilcoxon sign rank; Fig. $1 G, H)$. In contrast, in animals that received sham injections, induced gamma-band power was reduced after OFC pairing episodes similar to the control condition $(n=16$ sites in two animals; $p>10^{-3}$, paired $t$ test; Fig. $1 G$ ). Thus, the changes in the A1 population response depend on OFC activation rather than being the result of stimulation of fibers of passage or antidromic activation of A1 (or other) neurons projecting to OFC as

B

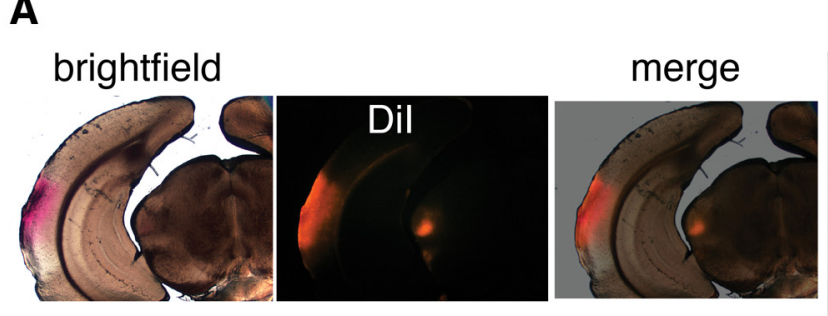

Fluo4

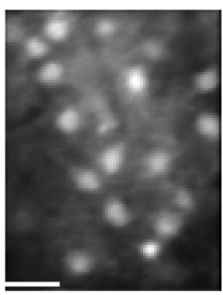

Tuning
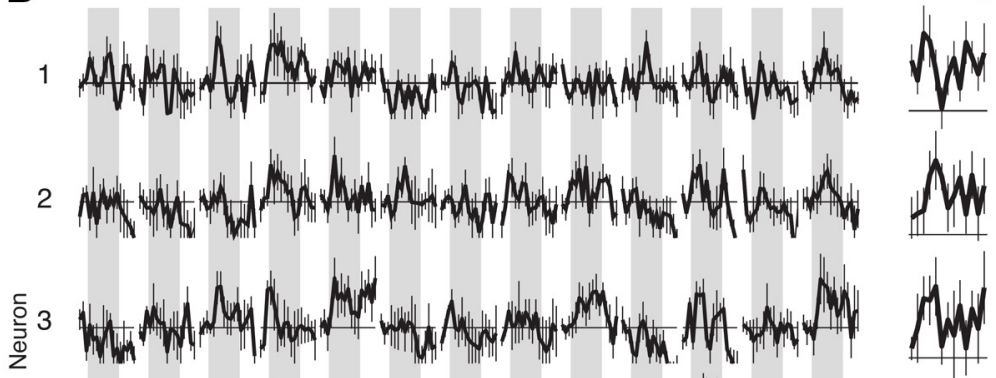

4 .

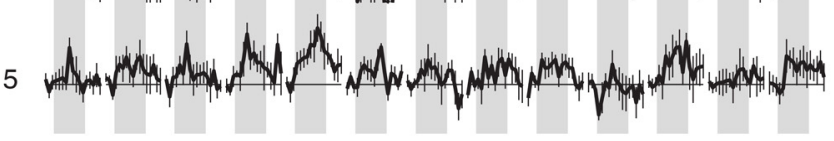

$L_{1 \mathrm{~s}}^{2 \% \Delta F / F}$
23

Frequency $(\mathrm{kHz})$

64
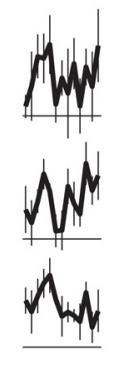

$8 \quad 64$

$(\mathrm{kHz})$
Figure 3. Plasticity of $\mathrm{A} 1$ neuron $B F$ with $\mathrm{OFC}$ microstimulation and sound pairing. $A$, Left, Anatomical identification of imaging ocation in A1. Right, Neurons are loaded with Fluo4-AM and $\mathrm{Ca}^{2+}$ responses are measured with two-photon imaging. $\boldsymbol{B}$, FluoNeurons denoted by circles; color: BF of neuron (see Materials and Methods). Color bar: frequency range. Right, BFs of the same cells after pairing with $23 \mathrm{kHz}$ (Post-pairing). Scale bars: $A, C, 25 \mu \mathrm{m}$.

these elements would be unaffected by pharmacological silencing of OFC neurons.

To confirm the effect of OFC on A1 responses we plotted the change in peak ERP amplitude as a function of sound frequency. We found that OFC pairing yielded frequency changes in LFP power. In particular we observed a decrease in responsiveness at frequencies flanking the PF (Fig. 1I). Silencing the OFC abolished these changes (Fig. 1I). Together our results show that OFC causes changes in the sound-evoked population response in A1.

\section{Cholinergic antagonists do not block OFC-induced desynchronization}

Our results demonstrate that OFC activity is necessary for altering A1 population activity. It is possible that the effects of stimulation-induced OFC signals on A1 population activity are mediated by a number of well known routes involving neuromodulatory centers and limbic areas that are engaged by OFC stimulation. To address this question, we assessed the contribu- 
A
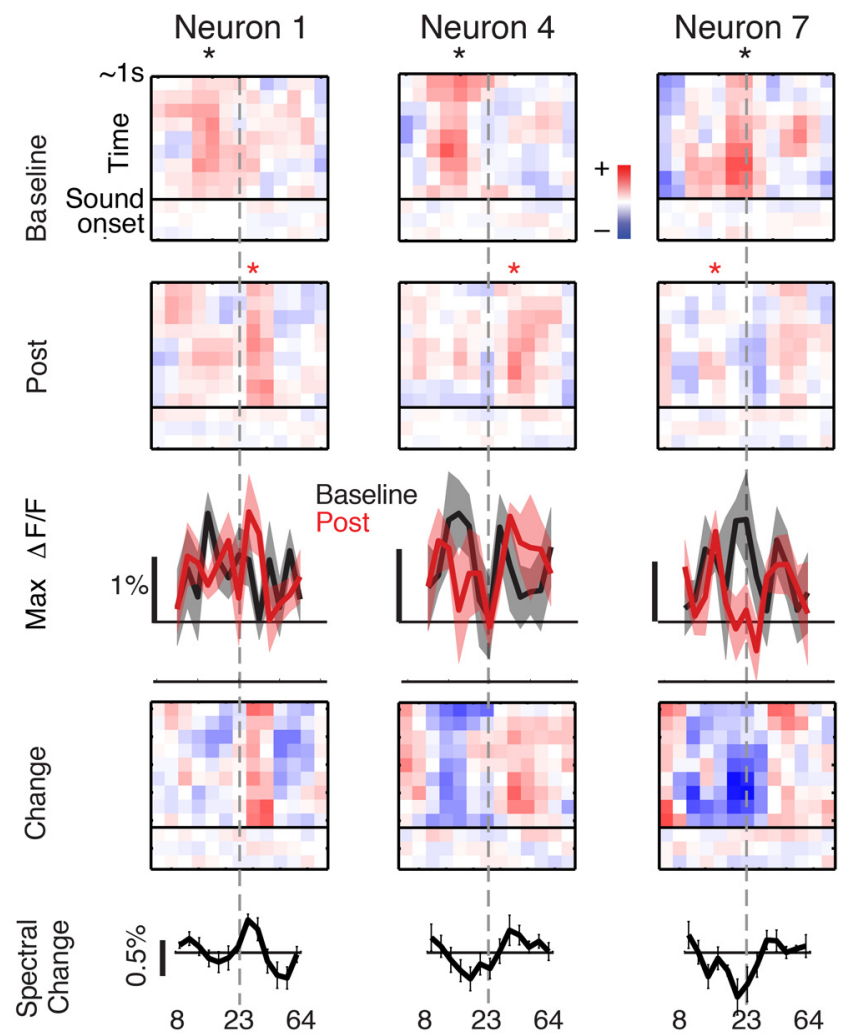

B
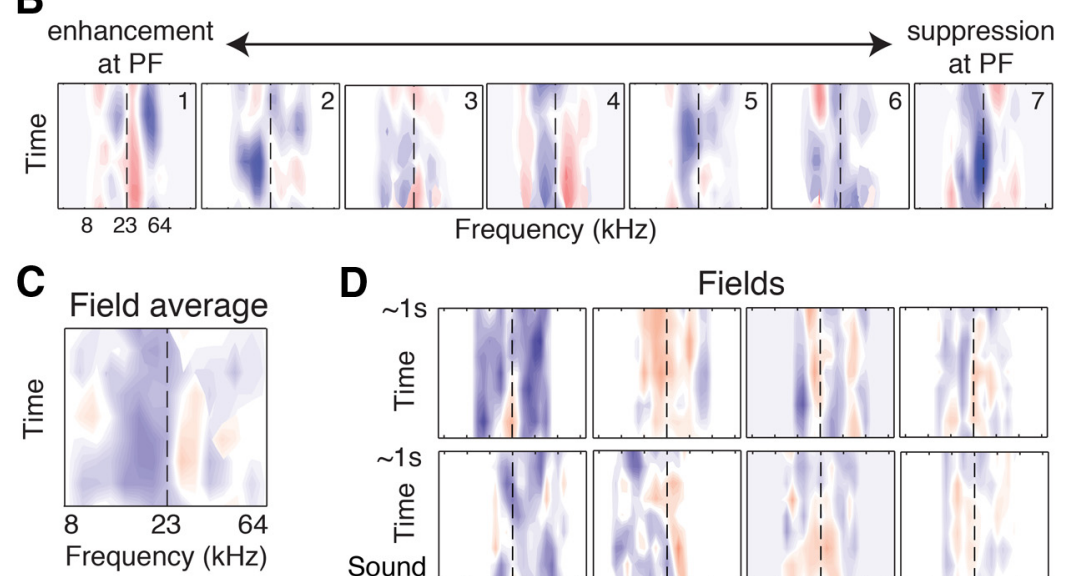

$\Delta \mathrm{F} / \mathrm{F}=+$

D
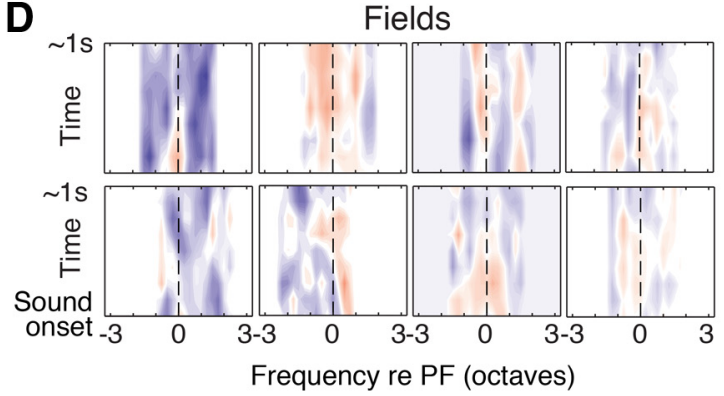

Figure 4. Plasticity of A1 neuron receptive fields with $\mathrm{OFC}$ microstimulation and sound pairing. $A$, Effects of OFC pairing paradigm on three representative neurons from the field in Figure 30). Each column represents data from a single neuron. Numbers are indicated in the field in Figure 3D). Abscissa: Frequency. Rows 1 and 2 represent smoothed $\mathrm{Ca}^{2+}$ responses before $0 \mathrm{FC}$ pairing (row 1 ) and after $0 \mathrm{FC}$ pairing (row 2). Color bar: $\Delta F / F( \pm 1 \%)$. Ordinate: Time from stimulus onset up to nine imaging frames later $(\sim 1 \mathrm{~s})$. Asterisks: BF before and after $\mathrm{OFC}$ pairing. Row 3 : Frequency tuning curves derived from maximum $\Delta F / F$ during the stimulus period. Black, before $0 \mathrm{FC}$ pairing (Baseline); Red, after OFC pairing; shading, $95 \%$ confidence intervals (see Materials and Methods). Ordinate: Max $\Delta F / F$. Row 4: Overall change in response (Post-Baseline) of the significant $(p<0.05) \Delta F / F$ responses for each neuron. For all rows: Dashed vertical line indicates PF. Row 5: Mean change over time showing the profile of spectral changes with $95 \%$ confidence interval. $\boldsymbol{B}$, Example time-frequency response differences for seven individual neurons indicated in Fig. 3C). Axes as in ( $A$ ) row 4 (1,4,and 7 same, now as contour plots). $C$, Mean change in response for all neurons in the field of view in $3 D$; color axis $\pm 0.5 \%$ ). D, Average DSTRMs after OFC pairing for 8 of 17 fields of view. Each plot shows the average difference (post-pre) in the responses of the neurons for each field of view. All DSTRMs are aligned to PF (dashed line). Scale is the same for all subplots as in $C$.

tion of ACh to the effects by measuring the effects of OFC-sound pairing episodes in the presence of antagonists for either muscarinic or nicotinic acetylcholine receptors (mAChRs or nAChRs, respectively). Similar to other studies of the effects of cholinergic signaling (Goard and Dan, 2009; Alitto and Dan, 2012) we first measured auditory responses to stimuli of various frequencies, topically applied antagonists (1 $\mathrm{mm}$ atropine, a selective $\mathrm{mAChR}$ antagonist, $n=3$ animals or $3 \mathrm{~mm}$ mecamylamine, a selective nAChR antagonist, $n=$ 3 animals) to the cortical surface for $>45$ min and assessed the effects of OFCsound pairing. In the presence of both drugs, we found gamma band-induced power at the PF was decreased after OFCpairing episodes (atropine median power: $2.57 \pm 1.35$ iqr; PostPair: $2.16 \pm 1.15$ iqr, $p<0.05$, meca: $1.96 \pm 0.93$ iqr; PostPair: $1.22 \pm 0.53$ iqr, $p<10^{-3}$; Wilcoxon sign rank; Fig. $2 A, B$ ). Thus, similar to control (Fig. $1 H$ ), OFC-pairing episodes caused significant desynchronization of the population responses in Al (Atropine: $-0.11 \pm 0.27$; mecamylamine: $-0.19 \pm$ 0.27 ; $p<0.03$; Wilcoxon sign rank; Fig. $2 C$ ). We next analyzed the frequency specificity of OFC activation after blocking cholinergic signaling. ERP amplitudes revealed frequency-specific changes similar to what was observed in the absence of atropine. The magnitude of the maximal change was larger in the absence of cholinergic signaling than under control conditions (Fig. 2D). Collectively, these results suggest that a large fraction of the changes in $\mathrm{A} 1$ induced by OFC pairing episodes is mediated by noncholinergic mechanisms.

\section{OFC-sound pairing changes the} frequency selectivity of A1 neurons Our electrophysiological results show a specific effect of OFC activation on the population activity of Al neurons. We next investigated how single neurons in A1 changed as a result of OFC activation. To simultaneously sample sound-evoked responses in many neurons we used in vivo two-photon $\mathrm{Ca}^{2+}$ imaging in $\mathrm{A} 1$ (Bandyopadhyay et al., 2010; Winkowski and Kanold, 2013). We confirmed location of the imaging site in A1 by retrograde DiI labeling of the MGB (Fig. 3A). We measured sound-evoked responses and frequency selectivity of $\mathrm{A} 1$ neurons in layer $2 / 3$ before and after pairing of tones with OFC stimulation using a range of isointensity SAM tones (Bandyopadhyay et al., 2010; Winkowski and Kanold, 2013). Before pairing, individual A1 neurons exhibited tone-evoked $\mathrm{Ca}^{2+}$ responses to a limited sound frequency range (Fig. $3 B$ ). Consistent with previous studies (Bandyopadhyay et al., 2010; Rothschild et al., 2010; Winkowski and Kanold, 2013), the observed distribution of BFs of A1 neurons within this field of view $(<100 \mu \mathrm{m})$ was broad (Fig. 3C, left). For the pairing episode, we 
selected a sound of a single frequency (tone) and paired that with electrical stimulation of the OFC. In each case, the $\mathrm{PF}$ was chosen to be within the range of BFs of the neurons within the imaged field. In the case shown (Fig. $3 C$ ), $23 \mathrm{kHz}$ was chosen as the $\mathrm{PF}$ ( 100 pairings, ISI $=$ $5 \mathrm{~s}, 200 \mu \mathrm{A}$ in OFC); in other cases, different sound frequencies were used as the choice of PF depended on the range of frequencies observed in the imaged area. After pairing, we measured sound-evoked responses again using the same range of tones as before pairing. We found that after pairing the BFs of many responding neurons changed (Fig. $3 C$, right). These results suggest a role of OFC activation in regulating plasticity within $\mathrm{A}$.

The $\mathrm{BF}$ of a neuron gives only limited information about the detailed changes that occurred to the neuron's frequency selectivity. To obtain a more complete picture of the effects of OFC pairing on the responses of A1 neurons, we plotted frequency tuning curves (Fig. $4 A$ ) as well as the sound-evoked responses across both frequency and time, or spectrotemporal response maps (STRMs), of neurons in the field before and after OFC pairing (Fig. 4A, rows 1 and 2, respectively). Before OFC pairing, sound-evoked $\mathrm{Ca}^{2+}$ responses of each neuron exhibited tuning for a range of frequencies (Fig. $4 A$, rows 1 and 3 ). After OFC pairing, the responses of neuron 1 to sound frequencies near the PF were enhanced (Fig. 4A, column 1, nonoverlapping 95\% confidence intervals); whereas, the responses of neuron 4 to sound frequencies slightly higher than the PF were enhanced (Fig. 4A, column 2). In neuron 7, responses to the PF were powerfully suppressed (Fig. 4A, column 3 ). These changes were visible in the differences in the STRMs of the neurons, which were computed by subtracting the STRM before pairing from the STRM after pairing (post-pre or DSTRM; Fig. 4A, row 4). In particular, the differential STRMs (DRSTM) indicate that changes were present throughout the duration of the tone presentation. The spectral profiles of the changes (mean change over time) indicate the frequency-specific enhancement or suppression (Fig. 4A, row 5). Based on the OFC-induced response changes of these three neurons as well as others within this imaged field, the observed changes in tuning in individual A1 neurons in the field were diverse (Fig. $4 B$ ). Despite this diversity in the OFC stimulation-induced changes in responsiveness, the most commonly observed change as indicated in the mean effect across all neurons in this field was suppression of responsiveness to sound frequencies below the PF and a modest enhancement of responsiveness just above the PF (Fig. 4C). Similar patterns of frequency-specific enhancement and suppression were seen at each imaging location even though different PFs were used at each location (Fig. 4D).

The average response change for the population of all imaged neurons ( $n=307$ neurons, 17 imaging locations, 6 animals, 3 stimulation levels: 100,200 , and $400 \mu \mathrm{A}$ ) revealed a consistent effect (Fig. 5A). Pairing OFC stimulation with a fixed-frequency sound caused facilitation of sound-evoked $\mathrm{Ca}^{2+}$ signals near the $\mathrm{PF}$ and suppression of sound-evoked $\mathrm{Ca}^{2+}$ signals for other
B

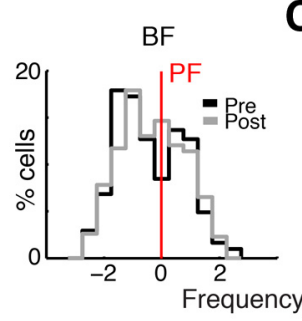

C

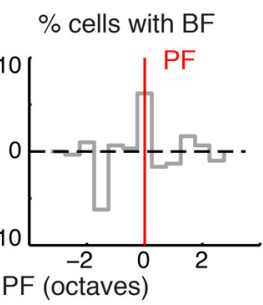

F mean change
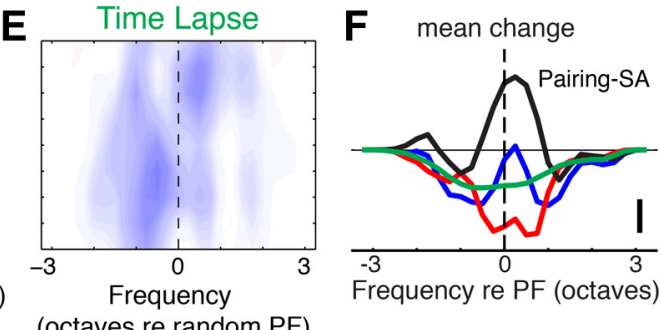

Frequency re PF (octaves)

(octaves re random $\mathrm{PF}$ )

Figure 5. Overall effect of OFC microstimulation and sound pairing in A1. $A$, Mean change in response for the population of imaged neurons that underwent $\mathrm{OFC}$ pairing. Color axis $\pm 0.1 \%$. B , Distribution of BFs of all neurons before (black) and after (gray) gefore pairing distribution shows that PFs were chosen such that different spectral distances (PF-BF) over \pm 3 octave over time showing spectral profile of changes in each case. The effective change in spectral profile due to the OFC pairing is shown in black after removing the effect of habituation caused by the presentation of a sound alone. Scale bar, $0.2 \%$.

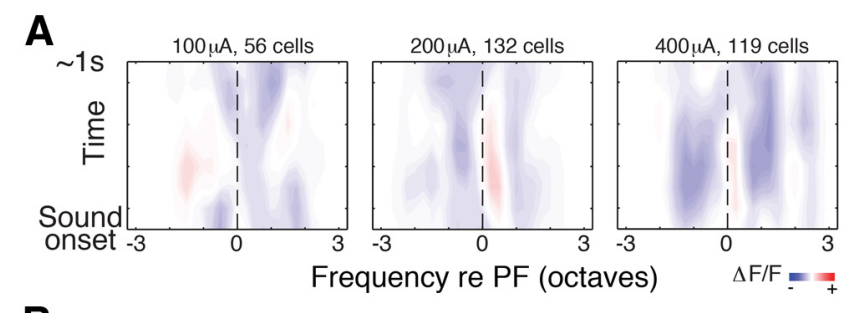

B

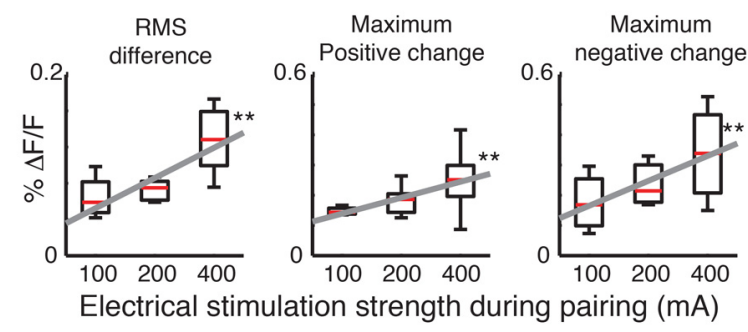

Figure 6. Effect OFC microstimulation and sound pairing in $\mathrm{A} 1$ depends on stimulation strength. $\boldsymbol{A}$, Mean changes in STRMs with OFC pairing in the population of neurons at different stimulation strengths. Strongest enhancements were observed at $P F$ at the highest stimulation strength and weak changes at the lowest stimulations strength. Axes as in Figure $3 A$ and $B$ (color bar $\pm 0.2 \%)$. $\boldsymbol{B}$, Measures of response change plotted as a function of $0 \mathrm{FC}$ stimulation strength, straight lines show the significant $\left({ }^{* *} p<0.05\right)$ best linear fits. All measures of magnitude of response change were positively correlated with $\mathrm{OFC}$ stimulation strength (Fig. 4).

sound frequencies within 1-1.5 octaves around the PF (Fig. 5A) and thus increased the numbers of neurons responding to the PF (Fig. $5 B, C$ ).

Frequency-specific enhancement of A1 responses depends on OFC-sound pairing

The changes in STRMs after pairing could be due to the OFC pairing episode or alternatively be a consequence of the animals 
A

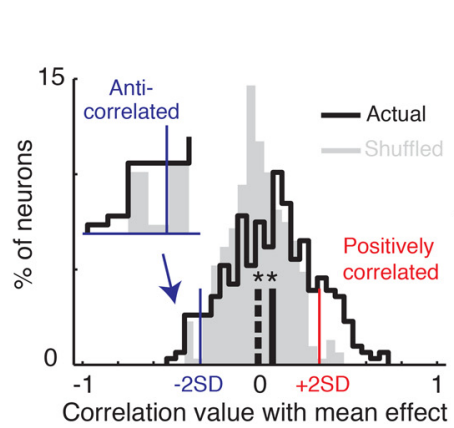

B

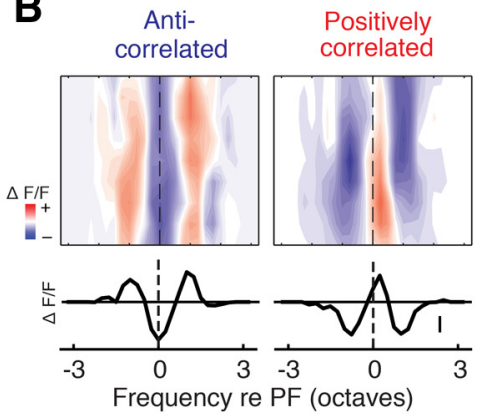

C

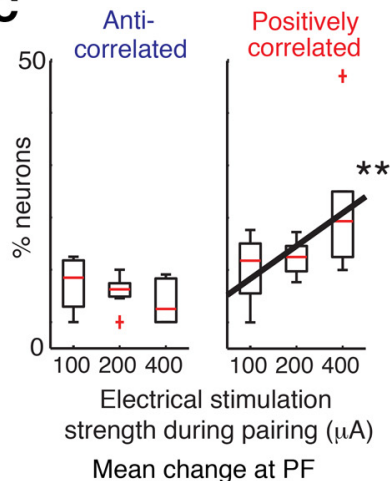

E

Mean change at PF
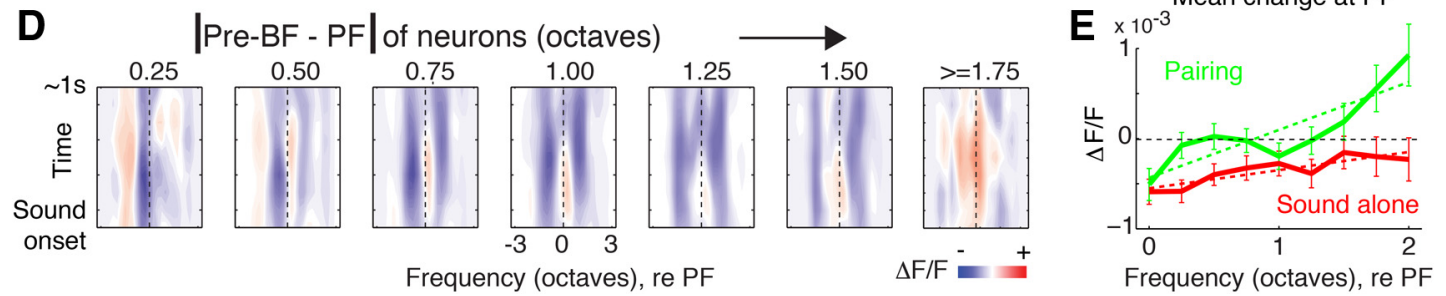

Figure 7. Two populations of response changes after OFC-sound pairing. $A$, Distributions of correlation values of actual response differences (after-before pairing) for each neuron with the mean effect shown in Figure $3 A$ (black line) and of shuffled version of the response differences of same neurons (gray). Solid and dashed vertical black lines: mean of the distributions of actual and shuffled datasets; red and blue lines: $\pm 2 *$ STD of shuffled data. Performing the same calculation but computing the correlation value of each neuron's response change with the average of all other neurons' response change produced similar results. Inset shows the increased number of neurons with correlations $<2$ STD. $\boldsymbol{B}$, Top, Average differences of the anticorrelated and positively correlated neurons (color axis $\pm 0.4 \%$ ). Bottom, Time-averaged mean spectral profiles of changes. $C$, Relationship of fraction of anticorrelated and positively correlated cells in each field as a function of stimulations strength. $D$, Mean response change in 0.5 octave bins around PF plotted as a function of spectral separation of before-BF from PF as changes are approximately symmetrical changes above and below PF. $E$, Mean $\Delta F / F$ change at PF and adjacent frequency bins as function of before-BF-PF for OFC pairing and sound alone. Dashed lines indicate regression lines. The slopes are significantly different $(p<0.05)$. Note that with sound alone no strengthening is observed.

experiencing a single sound repeatedly. To distinguish between these two possibilities we measured response changes produced by repeated presentation of a single frequency sound without pairing it with OFC activation (different sound frequencies were used in each imaging field, 100 repeats, ISI $=5 \mathrm{~s}$ ). In contrast to the enhancement seen after OFC pairing, responses near the repeated frequency were suppressed reflecting habituation (Condon and Weinberger, 1991; $n=209$ neurons; Fig. 5D). Thus, frequency-specific enhancement of A1 responses (Fig. 5A) requires OFC stimulation.

Neurons can show variable responses over time (Elhilali et al., 2007) and this variability can influence the observed OFC pairing-induced changes. The variability of sound-evoked $\mathrm{Ca}^{2+}$ responses over time measured with two-photon imaging has not been reported. To account for response variability induced by bleaching and by differences in optical sectioning over time, in addition to the sound-alone control experiment, we performed additional imaging experiments in which neural responses were measured twice to the same set of stimuli separated by a time gap of 5-10 min (equivalent to the pairing period, $n=3$ animals, 12 fields of view, 227 cells). To test if "spontaneous" changes in the time-lapse dataset could explain the observed enhancement at the $\mathrm{PF}$ in our pairing experiments, we analyzed the data in the same way as the pairing data (post-pre; see Materials and Methods) and aligned time-lapse changes in responses to a randomly assigned PF for each imaged field. We found the average effect (post-pre) to be a general depression at all frequencies without an enhancement at any specific frequency (Fig. 5E). This general depression resembles the effect of repeating a sound alone (Fig. $5 D$ ), but is smaller in magnitude (average $47 \%$ at PF, $p<0.001, t$ test) and might be due to a decrease in responsiveness over the imaging period as a result of bleaching or extrusion of the $\mathrm{Ca}^{2+}$ dye. Regardless, since there is no frequency bias to the average change in the time-lapse data (Fig. 5E), this depression cannot account for the changes that we observe with OFC pairing. Moreover, when accounting for habituation and time-dependent depression, the net effect of OFC pairing with a particular sound frequency shows a clear peaked enhancement of responses at and around PF in the spectral profile $\left(\mathrm{STRM}_{\text {Pairing }}-\mathrm{STRM}_{\text {SoundAlone; }}\right.$; Fig. 5F, black, assuming additive effects in the two conditions). Collectively, these results demonstrate that the frequencyspecific changes in A1 depend on OFC stimulation and the OFC pairing episode.

\section{Response change after OFC-sound pairing depends on stimulation strength}

Since OFC activity is thought to encode reward value and since activity of OFC neurons scales with the magnitude of reward (O'Doherty et al., 2003; Roesch and Olson, 2004; Wallis, 2007), we hypothesized that a larger signal originating in the OFC could lead to larger changes in sensory cortex to enhance the detection of a rewarding stimulus. We thus investigated whether the magnitude of $\mathrm{A} 1$ response modulation by OFC depended on the strength of OFC activation. We used three progressively larger OFC stimulation levels, which presumably activate a progressively larger fraction of the OFC and/or strengthens the activation of the same fraction. We found that the magnitude and nature of neuronal changes depended strongly on the level of activation of OFC during pairing (Fig. $6 A$ ), with the strongest enhancements at PF seen for the highest stimulation strength. Nearly all measures quantifying the magnitude and nature of response changes were significantly positively correlated with OFC stimulation strength (Fig. 6B). Thus, the facilitation of responses in $\mathrm{A} 1$ to sounds near the $\mathrm{PF}$ is directly dependent on the strength of OFC activation sug- 
gesting a mechanism by which higher reward value might lead to larger changes in sensory cortices.

\section{Neurons show enhancement or suppression at PF following OFC-sound pairing}

To test if the spectrotemporal structure of the average response change produced by OFC pairing was significant, we generated "shuffled" versions of the changes for each neuron (methods) and compared each of them to the mean effect. This generated a distribution of correlation values centered on zero (Fig. $7 \mathrm{~A}$, gray distribution, mean: black dashed line), whereas the same comparison of the "actual" changes for each neuron with the mean effect resulted in a distribution that was significantly shifted in the positive direction (mean: black line; asterisks: $p<10^{-5} t$ test), indicating that, on average, the change in neural response for individual neurons resembled the mean change. There were three times as many cells that exhibited significant response enhancement than suppression near PF. Specifically, $16 \%$ of the imaged neurons (48/307) exhibited significant positive correlations with the mean effect for the population (Fig. $7 A$, right of red bar), while $5 \%$ were significantly anticorrelated (16/307; Fig. $7 A$, left of blue bar). Thus, the mean change in STRM of the two populations indicated that positively correlated neurons show response enhancement at the $\mathrm{PF}$, whereas anticorrelated neurons show response suppression at $\mathrm{PF}$ (Fig. $7 B$ ).

The differential effects of OFC pairing in the two groups may depend on OFC stimulation strength. Indeed, plotting the fraction of neurons that were positively correlated with the mean effect showed that with increasing OFC stimulation strength a larger fraction of neurons were positively correlated (Fig. 7C). In contrast, while the anticorrelated neurons exhibited a negative trend, the effect did not reach significance $(p>0.05)$. This indicates that different cells in A1 might be engaged differentially depending on the strength of OFC activation.

The differential effects in these two groups of neurons may depend on the relative spatial position of the neurons within the cortex. However, we found that neurons with positive and anticorrelated effects were spatially intermingled (data not shown) consistent with the heterogeneous spatial organization of the superficial layers of mouse A1 (Bandyopadhyay et al., 2010; Rothschild et al., 2010; Winkowski and Kanold, 2013). Alternatively, the two populations of change could depend on the relative separation of the $\mathrm{PF}$ and the $\mathrm{BF}$ of the neuron before OFC pairing. Indeed, neurons with BFs near ( $<0.5$ octave) the PF tended to be anticorrelated with the mean effect, while neurons with BFs further away $(>0.5$ octave) from the PF tended to be positively correlated with the mean effect (Fig. $7 D, E$ ). Thus, the direction and magnitude of the OFC pairing-induced response change depended on the spectral separation of the neuron's BF before OFC pairing and the PF $(p<0.003$; Fig. $7 D, E)$. To test if the emergence of two cell populations was dependent on OFC pairing we analyzed the time-lapse data in the similar manner. There was no difference between the actual and shuffled groups for the timelapse data (Fig. 8; Kolmogorov-Smirnov test, $p>0.44$ ) indicating a specific effect of OFC pairing on the STRMs. Together, these results indicate that the form of A1 plasticity described here depends on the OFC pairing episode and the relation of the PF to the $\mathrm{BF}$ of each neuron.

\section{Differential effects of OFC-sound pairing episodes on correlated neuronal activity}

Our electrophysiological results show an effect of OFC activation on population activity in A1. In particular our electrophysiolog-

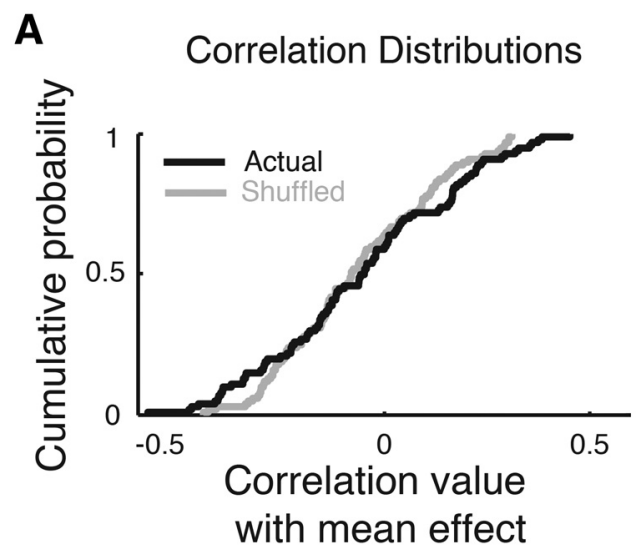

B Time-lapse change in BF

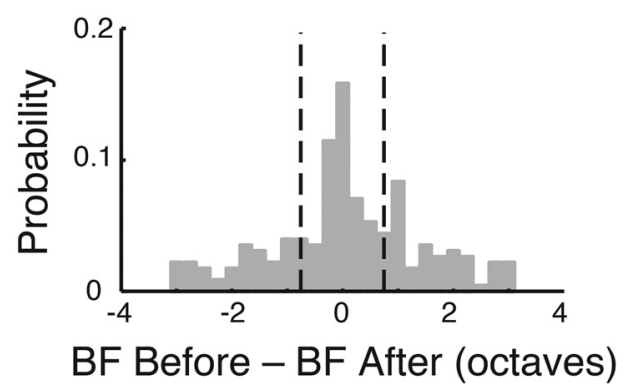

Figure 8. Time-lapse data show no specific changes. $\boldsymbol{A}$, Cumulative distribution of correlation values of time-lapse response changes with the mean effect shown in Figure $3 A$. Distributions were generated by selecting a random PF for each imaged field (repeating 100 times for each imaged field) and aligning response changes according to the PF, then averaging. Response changes were also shuffled in frequency and time to create a shuffled distribution. Cumulative distribution functions are shown for correlation values with mean effect of actual differences (black) and shuffled differences (gray). This shows a lack of structure related to PF in the time-lapse data, with changes resembling random noise ( $p>0.44$, Kolmogorov-Smirnov test). $\boldsymbol{B}$, Time-lapse changes in BF of $A 1$ neurons. Distribution of changes in best frequency (BFBefore-BFAfter) in two sequential imaging sessions separated by $\sim 10 \mathrm{~min}$. Fifty percent of neurons exhibit stable BF (within 0.75 octaves; dashed lines). The remaining $50 \%$ of imaged neurons had altered BF. With single-unit recordings in awake ferrets such changes measured for only linear cells with good spectrotemporal receptive fields (STRFs) find $>25 \%$ of cells are labile (Elhilali et al., 2007).

ical results suggest a decorrelation of neuronal responses and our imaging shows that OFC-sound pairing causes changes on the level of single neurons. How then do correlations between pairs of neurons change as a result of OFC stimulation? Despite the local heterogeneity of A 1 responses in layers 2/3 (Bandyopadhyay et al., 2010; Rothschild et al., 2010; Winkowski and Kanold, 2013), there are nevertheless many similarly responding neurons. Such redundancy may lead to decreased stimulus information in the neuronal population.

To investigate the correlated activity between pairs of neurons and how the changes described above influence neural encoding at the population level, we computed cross-correlations between mean responses of pairs of neurons (signal correlations) and trial-by-trial correlations in response variability (noise correlations) in each imaged field before and after each experimental condition (i.e., OFC pairing and sound alone). In all baseline conditions (i.e., before OFC pairing, before sound alone), we found low but significant signal correlations between pairs of neurons (Rothschild et al., 2010; Winkowski and Kanold, 2013; Fig. $9 A$; before OFC pairing: $0.10 \pm 0.15$ ). These low values were expected based on the weak similarity of neural responses in our 
A Signal correlations
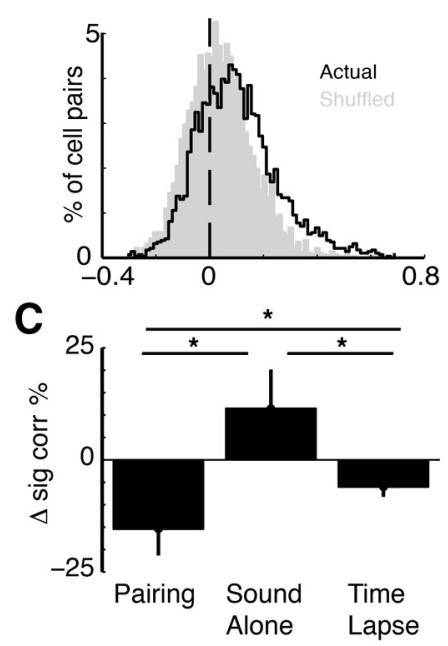

D
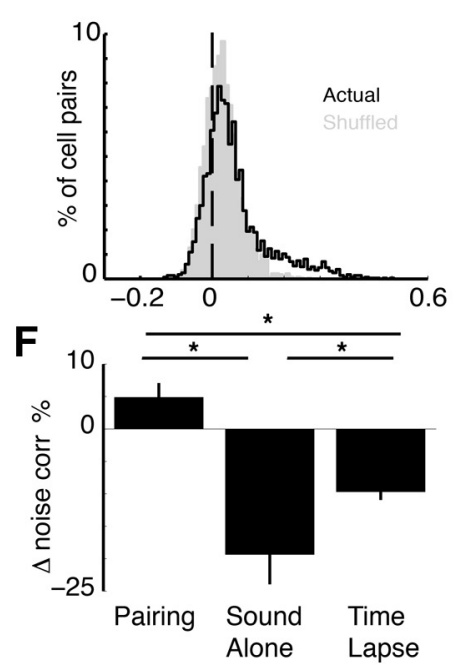

B

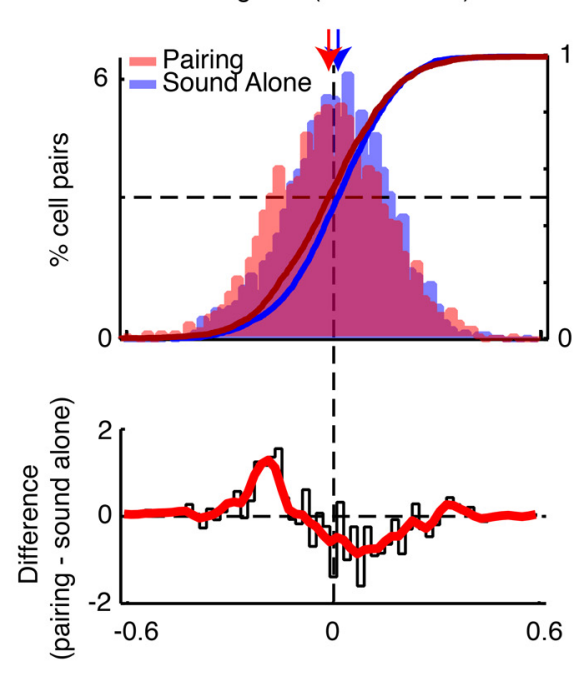

E

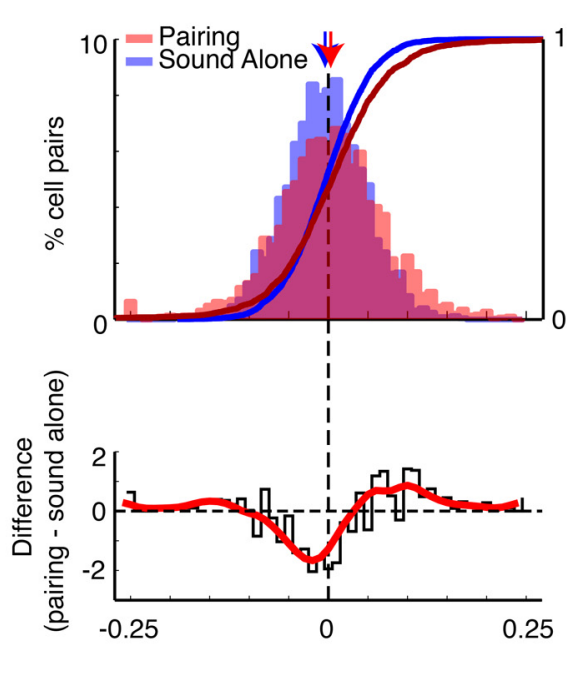

Figure 9. Changes in pairwise correlations between neurons produced by OFC pairing and sound alone. $A$, Distribution of pairwise signal correlation values between all simultaneously imaged pairs before OFC pairing (black). Distribution of correlation values of pairwise signal correlation values of frequency and time shuffled STRMs (gray). $\boldsymbol{B}$, Top, Distributions of changes in pairwise signal cross-correlations between all simultaneously imaged pairs of neurons in A1. Red, OFC pairing condition; blue, sound alone condition. Overlap between the distributions is shown in purple. Cumulative distribution functions are overlaid (red, OFC pairing; blue: sound alone). Bottom, Difference between the two distributions on the top part (Pairing - sound alone). Black line indicates raw difference; red line indicates smoothed version of the difference. $C$, Fractional change in mean correlations before and after OFC pairing show a significant decorrelation relative to zero $\left(p<10^{-6}\right)$ and the time-lapse case $(p<0.01)$ while that for sound alone showed a significant increase in mean correlations relative to zero $(p<0.01)$ and the time-lapse case $(p<$ 0.001). D, Distribution of pairwise noise correlation values between all simultaneously imaged pairs before OFC pairing (black). Distribution of correlation values of pairwise noise correlation values of frequency and time-shuffled STRMs (gray). $\boldsymbol{E}, \boldsymbol{F}$, Conventions as in $\boldsymbol{B}$ and $\boldsymbol{C}$, showing equivalent plots for noise correlations between pairs of neurons. Fractional change in mean noise correlations before and after $\mathrm{OFC}$ pairing shows a significant increase relative to zero $(p<0.05)$ and time-lapse case $\left(p<10^{-10}\right)$ while that for sound alone showed a significant decrease from $0\left(p<10^{-4}\right)$ and from the time-lapse case $(p<0.05)$.

data. To study how signals originating in the OFC influenced this correlated activity, we calculated the change in the signal crosscorrelations within each condition (after-before;(Fig. 9B, Pairing: red distribution; Sound alone: blue distribution). After OFC pairing, pairwise signal cross-correlations decreased significantly (Fig. $9 B, C ; p<10^{-6}$, paired $t$ test). In contrast, after repeating a sound without OFC activation (sound alone), pairwise correlations increased significantly (Fig. $9 B, C ; p<0.01$, paired $t$ test). In addition, the same analyses on the control time-lapse data showed an overall small decrease in signal correlations $(\sim 5 \%)$, which was expected due to decreases in responses over time (Fig. 9C). Thus, OFC pairing episodes resulted in a significant decrease in correlated stimulus-driven activity whereas sound alone episodes resulted in a significant increase in correlated stimulusdriven activity. Together, these processes are likely to have a powerful influence on neural coding within A1.

Since response similarity alone does not determine coding capacity as it also depends on correlations in response variability, we investigated whether the OFC pairing episodes had any influence over noise correlations (Fig. 9D). After OFC pairing episodes, noise correlations increased significantly (Fig. $9 E, F, p<0.05$, paired $t$ test). In contrast, after repeating a sound without OFC activation (sound alone), noise correlations decreased significantly (Fig. 9E, F, $p<10^{-4}$, paired $t$ test). In addition, the same analyses on the control time-lapse data showed a small decrease in noise correlations. The differential effects on signal and noise correlations after OFC pairing, and the opposite trend in the control datasets, lead, in principle, to an increase in coding capacity within A1 networks.

\section{OFC-sound pairing increases $\mathrm{PF}$ stimulus discriminability}

The differential changes of signal and noise correlations can lead to improved discrimination performance by neural populations. To test this idea directly, we calculated discrimination performance for population of neurons using a symmetrical Kullback-Leibler divergence (essentially $d^{\prime 2}$, with a Gaussian distribution assumption; see Materials and Methods). We calculated average discrimination performance of neural populations of varying sizes ( $N=1-14$ neurons) in two groups (PF/O: PF and any other frequency; O/O: two other stimulus frequencies neither of which was the PF) before and after each experimental condition (OFC pairing, sound alone, time-lapse). Significance and reliability of the discrimination calculations was assessed through computing discrimination based on baseline $\Delta F / F$ (no stimulus) and actual stimulus-driven $\Delta F / F$ responses (Fig. 10A,B). For the single cell case, the overall median discrimination performance was similar across experimental conditions (OFC pairing, sound alone, time-lapse) and discrimination groups (PF/O and O/O; Fig. 10C,D). Next, we investigated the effect of increasing population size on discrimination performance in both groups. Since Kullback-Leibler distances are biased (Johnson et al., 2001), we estimated bias in our distance metrics in two ways (see Materials and Methods) and find that 
bias is minimal ( $<2 \%$ of actual values; Fig. 11A). As population size increased, $\mathrm{PF} / \mathrm{O}$ discrimination performance improved significantly in both the OFC pairing and sound alone conditions (Fig. 11A). When we compared the actual improvement across conditions (OFC pairing, sound alone, time-lapse), we found $\mathrm{PF} / \mathrm{O}$ discrimination performance in the sound alone condition was higher than in the other conditions (OFC pairing condition and time-lapse; Fig. $11 B$, left). In contrast, $\mathrm{O} / \mathrm{O}$ discrimination performance worsened significantly in the OFC pairing condition but remained relatively unchanged in the sound alone condition (Fig. $11 B$, right, green and red dashed lines, respectively). When we calculated the "relative" changes in discrimination performance ([Post-Pre]/Pre; Fig. 11C) we found that as the population size increases, $\mathrm{PF} / \mathrm{O}$ discrimination performance improves in the OFC pairing condition but remains relatively flat (i.e., no improvement) in the sound alone condition. Thus, the differential effects on the $\mathrm{PF} / \mathrm{O}$ and $\mathrm{O} / \mathrm{O}$ discrimination performance in the OFC pairing condition and the similar effects across those same groups ( $\mathrm{PF} / \mathrm{O}$ vs $\mathrm{O} / \mathrm{O})$ in the sound alone condition result in a frequency-specific improvement in discrimination performance after OFC pairing that was not present in the sound alone condition.

Discrimination performance increases linearly with increases in population size, if one ignores interactions (i.e., correlations) between neurons. Clearly, these interactions cannot be ignored, and so, to examine only the contributions of correlations between neurons to discrimination performance as a function of population size, we considered normalized discrimination performance (see Materials and Methods). All discrimination performances were normalized by the discrimination performance of the corresponding single cell case. Thus for $N$ cells a normalized discrimination greater than $N$ would mean improvement over the independent case (Fig. $11 D$, black line), indicating a contribution of correlations between neurons to discrimination performance (indicated as $\Delta$ ). Indeed, this expected improvement occurs in both the OFC pairing and sound alone conditions (Fig. $11 D, E$ ). The resulting normalized discriminations (Pre and Post; Fig. $11 D, E$ ) show that following OFC pairing there is a strong improvement in discrimination performance that is due to the change in correlations; such an improvement was absent for the sound alone case. In addition, correlations did not contribute strongly for the other groups $(\mathrm{O} / \mathrm{O})$. Thus, the changes in $\mathrm{A} 1$ responses produced by the OFC pairing episodes cause improvements in discriminability of a specific stimulus, the PF, and correlations between neurons play a large role in this stimulus-specific improvement. Further, while single neurons, on average, do not show improvement in discrimination of $\mathrm{PF}$ for both OFC pairing and sound alone conditions by including the correlations between neu-

\section{B \\ PF/O Discrimination \\ (before)}

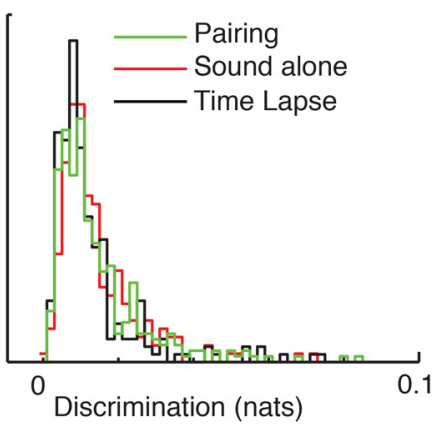

D Discrimination by single neurons

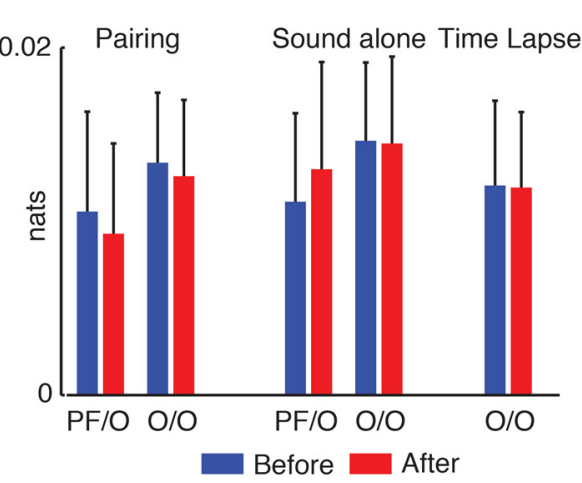

Figure 10. Discrimination of PF by single neurons. $\boldsymbol{A}$, Distribution of mean discrimination performance of $\mathrm{PF}$ from each other frequen (PF/0) by all neurons before OFC pairing (black). The same distribution based on the calculation done on baseline activity performance before pairing. $\boldsymbol{D}$, Bar graph showing median single neuron discrimination performance. Error bars indicate interquartile range. All groups are statistically the same $(p>0.1)$.

rons, we see a marked improvement in discrimination following OFC pairing. These results suggest that OFC pairing increases the discrimination ability of $\mathrm{A} 1$ in a frequency-specific manner.

\section{A limited number of patterns underlie OFC-induced response changes}

The spectrotemporal changes in sound evoked responses after OFC pairing were diverse (Figs. 3, 4). This diversity in response change could be due to any number of factors such as differences in cell type and/or the variability of the cells' responses to sound. Thus, to reveal the significant underlying patterns of changes that OFC pairing paradigm could produce and further explore the diversity of response changes in individual A1 neurons, we used principal components analysis (PCA) on the response changes for individual neurons to show distinct components of response changes within the population (Fig. 12). PCA can reveal the internal structure of the data in a way that best explains its variance. For the OFC pairing data, the first three principal components (PCs) that emerge from this analytical approach account for a large portion $(\sim 70 \%)$ of the variance and thus account for the response changes that are frequently observed in our population of imaged neurons (Fig. 12A), whereas each of the remaining components explain $<5 \%$ of the variance. Inspection of the centroids of the three dominant components revealed distinct patterns of change (Fig. 12B), with the first component (PC1) 
A

\section{Discrimination}

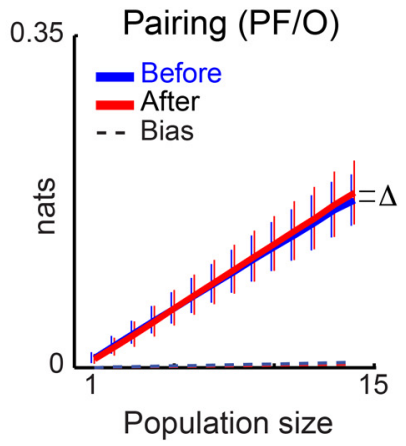

B
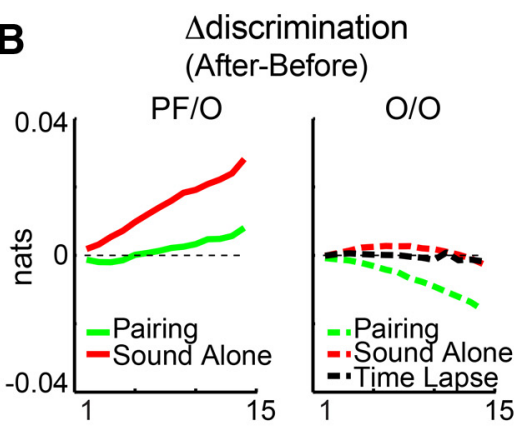

Population Size

D

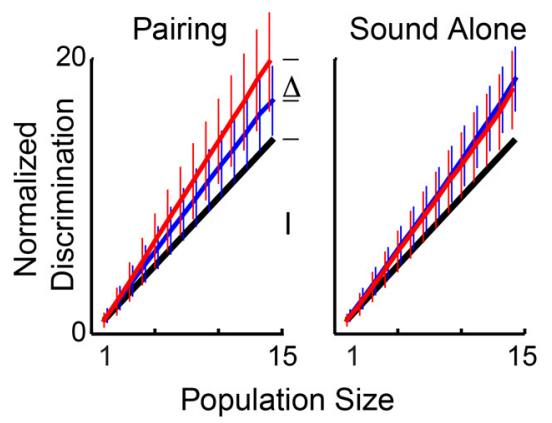

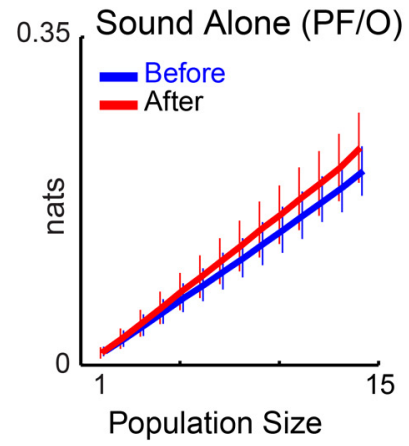

C $\% \Delta$ discrimination (After-Before)/Before

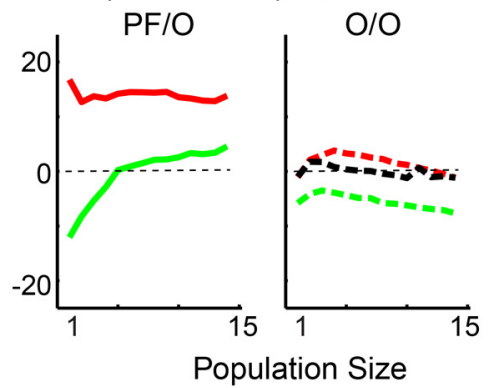

Population Size

E $\Delta$ Discrimination over independent neurons $(\Delta / \mathrm{l})$

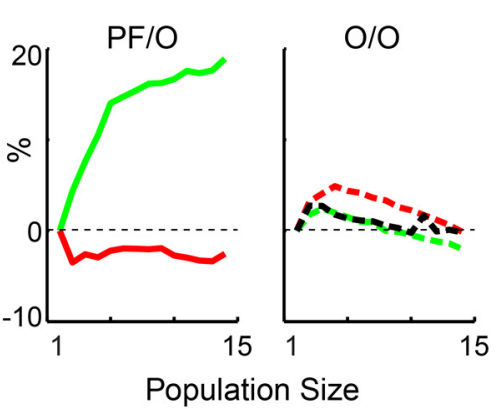

Figure 11. Discrimination performance as a function of population size. $A$, Median performance by populations of cells as a function of population size $(N=1-14)$ in discriminating PF from other frequencies before (blue lines) and after (red lines) OFC pairing (left) and sound presentation alone (right). Error bars indicate interquartile ranges. Data show no bias. $B, C$, Actual change (Post-Pre, left) $(\boldsymbol{B})$ and relative change $(\boldsymbol{C})$ ([Post-Pre]/Pre, right) in discrimination. Changes at the largest population size were always significantly different $(p<0.05)$. $\boldsymbol{D}$, Normalized discrimination (normalized to $N=1$ ) as a function of population size is shown for pairing and sound alone as in $A$, black line indicates discrimination by equivalent populations assuming independence between cells. $E$, Relative changes in discrimination due to correlations as a function of population size (changes are significant for $N=14, p<0.05)$ over the independent case.

showing enhancement at PF (Fig. 12B, arrow, bottom row) compared with suppression in side frequencies for the other PCA components. As is the case with PCA, the position of a variable along the component axis can be in the positive or negative direction and so the grayscale deviations (black or white) from the background of no change (gray) displayed in Figure $12 B$ can be interpreted to be changes that reflect either enhancement or suppression. The first and dominant pattern (Fig. 12B, left) of change is characterized by a relatively small amount of enhancement (or suppression) centered at the PF flanked by suppression (or enhancement) of responses to other sound frequencies, similar to the mean effect (Fig. 5). The second and third patterns, which account for lesser variability in the $\Delta$ STRMs, modulate the first pattern by imposing their shapes. The second pattern (Fig. 12B, middle) added to the first pattern tends to make the changes asymmetric around PF leading to a slope change at PF. The third pattern (Fig. 12B, right) modulates the first pattern by making the range of enhancement or suppression around the PF narrower or wider. Indeed, changes similar to each of the scenarios described above are observed in the examples shown in Figure 4 (neurons 1, 4, and 7). Furthermore, all patterns exhibit features that are related to the PF highlighting the frequency-specific nature of the observed OFC-induced effects. Increasing OFC stimulation current strength caused increases in the absolute distance of the response change for neurons from the origin (Fig. 12C). Such dependence on the stimulation strength shows that the diversity of response changes (at least $70 \%$ of the variance) cannot be explained by innate variability in the data. Thus, the OFC pairing paradigm causes different types of change in response patterns in Al neurons that are all specific for the PF. The existence of distinct patterns of response change likely reflects the differential engagement of distinct circuits or plasticity mechanisms within A1 by OFC activation, which, in turn, could account for the different types of changes observed at the single cell level.

\section{Discussion}

Here we have demonstrated that signals originating in one area of the mouse FC, the OFC, can cause changes on the level of single neurons and across populations of A1 neurons. We find that OFC signals cause dynamic reshaping of response properties of populations of sensory neurons in A1 over rapid timescales. Electrophysiological recordings showed that pairing OFC activation with sound caused rapid decorrelation of A1 neurons and that OFC activity is required for these changes to occur. Moreover, our pharmacological experiments suggest that the OFC pairing-induced decorrelation of A1 activity is largely mediated by noncholinergic mechanism. In addition, by using in vivo two-photon $\mathrm{Ca}^{2+}$ imaging to measure the OFC-induced response changes, we have provided a more complete picture of the population dynamics that occur within sensory neural networks as a result of our OFCsound pairing paradigm. Pairing OFC activation with a sound caused changes in the frequency selectivity of A1 neurons within minutes. Thus our results show that signals originating in the $\mathrm{OFC}$ can generate specific response changes in A1.

While OFC stimulation caused a robust effect on $\mathrm{A} 1$ responses detectable on the LFP level, the observed response changes at the level of single neurons were diverse and may be explained by at least three nonexclusive factors. First, the observed diversity in response change may be a consequence of the local heterogeneity 


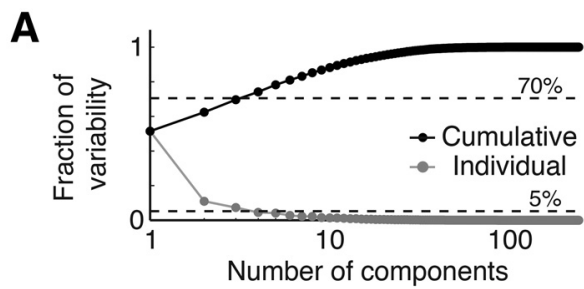

B
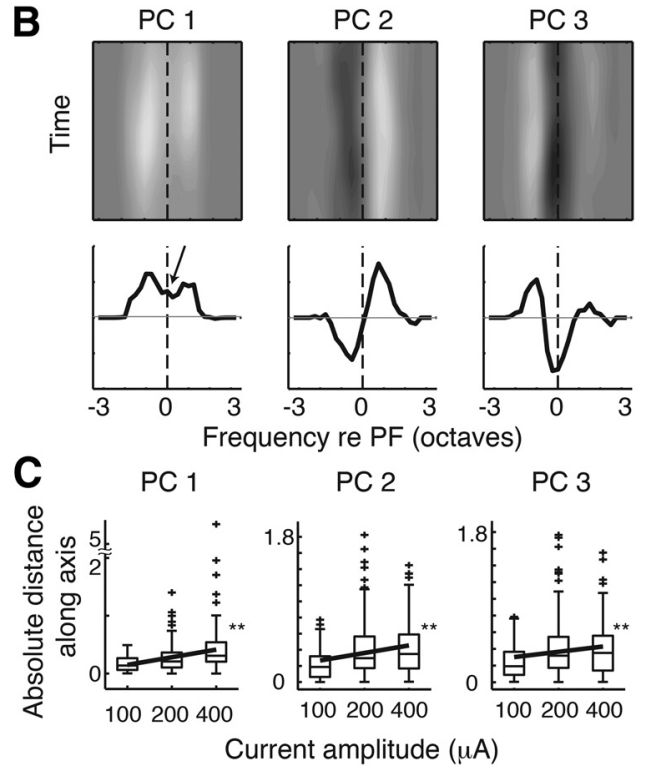

Figure 12. OFC sound pairing produces distinct types of response changes in $\mathrm{A} 1$ neurons. $\boldsymbol{A}$, Fraction of variability of the observed STRM changes with $0 F C$ pairing explained by each $P C$ and cumulatively starting from the strongest component shows that the first three PCs explain $70 \%$ of the variance, while each of the remaining explain $<5 \%$ of the variance. $B$, Patterns of underlying response changes as defined by the first three $P C s$. The mean spectral profile of each $P C$ is shown below. $C$, Absolute $P C$ scores or distance of response change along each $P C$ axis depends on OFC stimulation strength ( $p<0.0001,0.001$, and 0.05 , respectively, left to right).

of response properties in layer 2/3 of A1 (Bandyopadhyay et al., 2010; Rothschild et al., 2010; Winkowski and Kanold, 2013). Second, the diversity of OFC-induced changes in single A1 neurons may be the result of engagement of different pathways from OFC to A1. Thirdly, the observed diversity in response change could be due to the varied influences on different classes of A1 neurons. The presence of a small number (three) of distinct change patterns and the dependence of the change on the separation between PF and BF suggest that a combination of these factors might be responsible. The overall average outcome of the OFC pairing episode was to enhance the responsiveness of A1 neurons to the pairing frequency, which points to the OFC as a higher order cortical area capable of regulating responsiveness and organization within the auditory cortex. In addition, the correlation of the magnitudes of mean response change and the patterns of response change (Figs. 7, 12) with OFC stimulation strength suggest that the diversity of response changes could not be accounted for by activation of different pathways at different OFC stimulation intensities but likely reflect a stronger activation of similar pathways. Unfortunately, the precise identity of those pathways at this time remains unclear. However, we speculate that the OFC effects are likely to involve a highly complex interaction across multiple pathways including both direct and indirect connections between $\mathrm{OFC}$ and $\mathrm{A} 1$ and it is possible that differing behavioral demands activate different pathways possibly by activating distinct subsets of OFC neurons or modulating overall OFC activity levels. While our stimulation experiments here do not allow us to investigate these possibilities, future studies investigating OFC activity in various auditory behaviors should be able to distinguish between these possibilities.

Our data here indicate that a large fraction of the OFC activity-induced changes in A1 involves noncholinergic pathways such as direct corticocortical projections (Golmayo et al., 2003). The existence of different pathways might enable both rapid and long-lasting synaptic changes. For example, direct intracortical pathways could cause synaptic changes via short-term synaptic plasticity mechanisms that could be reinforced via indirect neuromodulatory pathways on longer timescales. The advantage of such a multipathway system is that it allows adaptations to rapidly changing environments by first "tagging" synapses and by later converting this transient change into longer lasting synaptic changes.

The role of the OFC in guiding behavior based on reward signals or expected outcomes is well documented (Schoenbaum et al., 2009). Damage to the OFC in humans leads to impulsivity, perseveration, compulsive behavior (Fellows, 2007), and impaired decision-making ability (Bechara et al., 1994, 1999) among other deficits. OFC lesions in animals have been reported to impair reversal learning (Burke et al., 2009; Takahashi et al., 2009), associative-learning (McDannald et al., 2005), and decision making, and to induce compulsive behavior (Schilman et al., 2010). OFC activity might also encode expected outcomes in reward prediction as well as reward value (Wallis, 2007). Collectively, these findings demonstrate that the OFC has a prominent role in facilitating flexible and adaptive behavior. When these findings are placed in the context of our current A1 plasticity results, it is plausible that some of the behavioral effects of OFC activity are due to response changes in primary sensory areas. The changes we observe after OFC-sound pairing are strikingly similar to the influence of sound discrimination behavior on auditory responses in ferret A1 (Fritz et al., 2003, 2007a) and the ferret FC is active during such sound discrimination behaviors (Fritz et al., 2010). Together this suggests that the changes we observe here could underlie the behavioral changes in A1. However, since the activation of the mouse OFC in auditory behaviors is unknown at this time, we can only speculate about the direct behavioral significance of A1 plasticity mediated by signals originating in OFC.

The animals used in our study are still relatively young (P20$\mathrm{P} 35)$. While this is beyond the critical period for changes in A1 (Barkat et al., 2011), and after the maturation of intrinsic circuits within frontal cortex (Uylings, 1990; Zhang, 2004; Yang et al., 2012) projections might not be fully mature. Given the profound effect we observe, we speculate that these might become stronger due to more mature and possibly stronger synaptic connections between OFC and A1. Moreover, degeneration or malformation of the OFC-A1 influence might play a role in cognitive decline with aging or underlie cognitive disorders such as autism (Funabiki et al., 2012; Orekhova et al., 2012).

Dynamic regulation of sensory cortical circuits by inputs originating in higher order cortical areas has been proposed as a mechanism by which animals successfully adapt to rapidly changing environments (Fritz et al., 2007a). The impact of OFC activation on auditory responses in A1 are strikingly similar to the influence of behavior in detection tasks on both auditory responses in A1 (Fritz et al., 2003, 2007a) as well as visual responses in visual cortical areas (Reynolds and Chelazzi, 2004; Cohen and Maunsell, 2009; Goard and Dan, 2009). Although the complete circuitry underlying top-down control of the auditory cortex is likely to be complex involving multiple projections and different 
neuromodulators and an array of different brain structures, our findings identify the OFC as a higher order prefrontal cortical region that can coordinate rapid, dynamic changes in response properties and functional organization within sensory cortical areas.

\section{References}

Alitto HJ, Dan Y (2012) Cell-type-specific modulation of neocortical activity by basal forebrain input. Front Syst Neurosci 6:79. Medline

Averbeck BB, Latham PE, Pouget A (2006) Neural correlations, population coding and computation. Nat Rev Neurosci 7:358-366. CrossRef Medline

Bakin JS, Weinberger NM (1996) Induction of a physiological memory in the cerebral cortex by stimulation of the nucleus basalis. Proc Natl Acad Sci U S A 93:11219-11224. CrossRef Medline

Bandyopadhyay S, Shamma SA, Kanold PO (2010) Dichotomy of functional organization in the mouse auditory cortex. Nat Neurosci 13:361368. CrossRef Medline

Bao S, Chan VT, Merzenich MM (2001) Cortical remodelling induced by activity of ventral tegmental dopamine neurons. Nature 412:79-83. CrossRef Medline

Barkat TR, Polley DB, Hensch TK (2011) A critical period for auditory thalamocortical connectivity. Nat Neurosci 14:1189-1194. CrossRef Medline

Bechara A, Damasio AR, Damasio H, Anderson SW (1994) Insensitivity to future consequences following damage to human prefrontal cortex. Cognition 50:7-15. CrossRef Medline

Bechara A, Damasio H, Damasio AR, Lee GP (1999) Different contributions of the human amygdala and ventromedial prefrontal cortex to decisionmaking. J Neurosci 19:5473-5481. Medline

Bokil H, Andrews P, Kulkarni JE, Mehta S, Mitra PP (2010) Chronux: a platform for analyzing neural signals. J Neurosci Methods 192:146-151. CrossRef Medline

Budinger E, Laszcz A, Lison H, Scheich H, Ohl FW (2008) Non-sensory cortical and subcortical connections of the primary auditory cortex in Mongolian gerbils: bottom-up and top-down processing of neuronal information via field AI. Brain Res 1220:2-32. CrossRef Medline

Burke KA, Takahashi YK, Correll J, Brown PL, Schoenbaum G (2009) Orbitofrontal inactivation impairs reversal of Pavlovian learning by interfering with 'disinhibition' of responding for previously unrewarded cues. Eur J Neurosci 30:1941-1946. CrossRef Medline

Cavada C, Compañy T, Tejedor J, Cruz-Rizzolo RJ, Reinoso-Suárez F (2000) The anatomical connections of the macaque monkey orbitofrontal cortex. A review. Cereb Cortex 10:220-242. CrossRef Medline

Chavez CM, McGaugh JL, Weinberger NM (2009) The basolateral amygdala modulates specific sensory memory representations in the cerebral cortex. Neurobiol Learn Mem 91:382-392. CrossRef Medline

Cohen MR, Kohn A (2011) Measuring and interpreting neuronal correlations. Nat Neurosci 14:811-819. CrossRef Medline

Cohen MR, Maunsell JH (2009) Attention improves performance primarily by reducing interneuronal correlations. Nat Neurosci 12:1594-1600. CrossRef Medline

Condon CD, Weinberger NM (1991) Habituation produces frequencyspecific plasticity of receptive fields in the auditory cortex. Behav Neurosci 105:416-430. CrossRef Medline

Cover TM, Thomas JA (2006) Elements of information theory. Hoboken, NJ: Wiley-Interscience.

Elhilali M, Fritz JB, Chi TS, Shamma SA (2007) Auditory cortical receptive fields: stable entities with plastic abilities. J Neurosci 27:10372-10382. CrossRef Medline

Fellows LK (2007) The role of orbitofrontal cortex in decision making: a component process account. Ann N Y Acad Sci 1121:421-430. CrossRef Medline

Fritz JB, Elhilali M, David SV, Shamma SA (2007a) Auditory attentionfocusing the searchlight on sound. Curr Opin Neurobiol 17:437-455. CrossRef Medline

Fritz JB, Elhilali M, Shamma SA (2007b) Adaptive changes in cortical receptive fields induced by attention to complex sounds. J Neurophysiol 98: 2337-2346. CrossRef Medline

Fritz JB, David SV, Radtke-Schuller S, Yin P, Shamma SA (2010) Adaptive, behaviorally gated, persistent encoding of task-relevant auditory infor- mation in ferret frontal cortex. Nat Neurosci 13:1011-1019. CrossRef Medline

Fritz J, Shamma S, Elhilali M, Klein D (2003) Rapid task-related plasticity of spectrotemporal receptive fields in primary auditory cortex. Nat Neurosci 6:1216-1223. CrossRef Medline

Froemke RC, Merzenich MM, Schreiner CE (2007) A synaptic memory trace for cortical receptive field plasticity. Nature 450:425-429. CrossRef Medline

Froemke RC, Carcea I, Barker AJ, Yuan K, Seybold BA, Martins AR, Zaika N, Bernstein H, Wachs M, Levis PA, Polley DB, Merzenich MM, Schreiner CE (2013) Long-term modification of cortical synapses improves sensory perception. Nat Neurosci 16:79-88. CrossRef Medline

Funabiki Y, Murai T, Toichi M (2012) Cortical activation during attention to sound in autism spectrum disorders. Research in developmental disabilities 33:518-524. CrossRef Medline

Ghashghaei HT, Barbas H (2002) Pathways for emotion: interactions of prefrontal and anterior temporal pathways in the amygdala of the rhesus monkey. Neuroscience 115:1261-1279. CrossRef Medline

Gilbert CD, Sigman M (2007) Brain States: top-down influences in sensory processing. Neuron 54:677-696. CrossRef Medline

Goard M, Dan Y (2009) Basal forebrain activation enhances cortical coding of natural scenes. Nat Neurosci 12:1444-1449. CrossRef Medline

Golmayo L, Nuñez A, Zaborszky L (2003) Electrophysiological evidence for the existence of a posterior cortical-prefrontal-basal forebrain circuitry in modulating sensory responses in visual and somatosensory rat cortical areas. Neuroscience 119:597-609. CrossRef Medline

Henrie JA, Shapley R (2005) LFP power spectra in V1 cortex: the graded effect of stimulus contrast. J Neurophysiol 94:479-490. CrossRef Medline

Johnson DH, Gruner CM, Baggerly K, Seshagiri C (2001) Informationtheoretic analysis of neural coding. J Comput Neurosci 10:47-69. CrossRef Medline

Josić K, Shea-Brown E, Doiron B, de la Rocha J (2009) Stimulus-dependent correlations and population codes. Neural Comput 21:2774-2804. CrossRef Medline

Kastner S, Pinsk MA (2004) Visual attention as a multilevel selection process. Cogn Affect Behav Neurosci 4:483-500. CrossRef Medline

Kilgard MP, Merzenich MM (1998) Cortical map reorganization enabled by nucleus basalis activity. Science 279:1714-1718. CrossRef Medline

McDannald MA, Saddoris MP, Gallagher M, Holland PC (2005) Lesions of orbitofrontal cortex impair rats' differential outcome expectancy learning but not conditioned stimulus-potentiated feeding. J Neurosci 25:46264632. CrossRef Medline

Meyer K (2011) Primary sensory cortices, top-down projections and conscious experience. Prog Neurobiol 94:408-417. CrossRef Medline

Miyashita T, Ichinohe N, Rockland KS (2007) Differential modes of termination of amygdalothalamic and amygdalocortical projections in the monkey. J Comp Neurol 502:309-324. CrossRef Medline

O'Doherty J, Critchley H, Deichmann R, Dolan RJ (2003) Dissociating valence of outcome from behavioral control in human orbital and ventral prefrontal cortices. J Neurosci 23:7931-7939. Medline

Orekhova EV, Tsetlin MM, Butorina AV, Novikova SI, Gratchev VV, Sokolov PA, Elam M, Stroganova TA (2012) Auditory cortex responses to clicks and sensory modulation difficulties in children with autism spectrum disorders (ASD). PLoS One 7:e39906. CrossRef Medline

Polley DB, Steinberg EE, Merzenich MM (2006) Perceptual learning directs auditory cortical map reorganization through top-down influences. J Neurosci 26:4970-4982. CrossRef Medline

Reynolds JH, Chelazzi L (2004) Attentional modulation of visual processing. Annu Rev Neurosci 27:611-647. CrossRef Medline

Roesch MR, Olson CR (2004) Neuronal activity related to reward value and motivation in primate frontal cortex. Science 304:307-310. CrossRef Medline

Rothschild G, Nelken I, Mizrahi A (2010) Functional organization and population dynamics in the mouse primary auditory cortex. Nat Neurosci 13:353-360. CrossRef Medline

Schilman EA, Klavir O, Winter C, Sohr R, Joel D (2010) The role of the striatum in compulsive behavior in intact and orbitofrontal-cortexlesioned rats: possible involvement of the serotonergic system. Neuropsychopharmacology 35:1026-1039. CrossRef Medline

Schoenbaum G, Roesch MR, Stalnaker TA, Takahashi YK (2009) A new 
perspective on the role of the orbitofrontal cortex in adaptive behaviour. Nat Rev Neurosci 10:885-892. Medline

Takahashi YK, Roesch MR, Stalnaker TA, Haney RZ, Calu DJ, Taylor AR, Burke KA, Schoenbaum G (2009) The orbitofrontal cortex and ventral tegmental area are necessary for learning from unexpected outcomes. Neuron 62:269-280. CrossRef Medline

Uylings HBM (1990) The Prefrontal cortex its structure, function, and pathology: proceedings of the 16th International Summer School of Brain Research, held at the Royal Tropical Institute and the Royal Netherlands Academy of Sciences Amsterdam, from 28 August to 1 September 1989. Progress in Brain Research, Vol. 85. Amsterdam: Elsevier.

Wallis JD (2007) Orbitofrontal cortex and its contribution to decisionmaking. Annu Rev Neurosci 30:31-56. CrossRef Medline
Weinberger NM (2007) Associative representational plasticity in the auditory cortex: a synthesis of two disciplines. Learn Mem 14:1-16. CrossRef Medline

Winkowski DE, Kanold PO (2013) Laminar transformation of frequency organization in auditory cortex. J Neurosci 33:1498-1508. CrossRef Medline

Yang JM, Zhang J, Yu YQ, Duan S, Li XM (2012) Postnatal development of 2 microcircuits involving fast-spiking interneurons in the mouse prefrontal cortex. Cereb Cortex. Retrieved October 4, 2012. Advance online publication. doi:10.1093/cercor/bhs291. CrossRef

Zhang ZW (2004) Maturation of layer V pyramidal neurons in the rat prefrontal cortex: intrinsic properties and synaptic function. J Neurophysiol 91:1171-1182. Medline 